\title{
Filling-enforced constraint on the quantized Hall conductivity on a periodic lattice
}

\author{
Yuan-Ming Lu, ${ }^{1}$ Ying Ran, ${ }^{2}$ and Masaki Oshikawa ${ }^{3,4}$ \\ ${ }^{1}$ Department of Physics, The Ohio State University, Columbus, Ohio 43210, USA \\ ${ }^{2}$ Department of Physics, Boston College, Chestnut Hill, MA 02467, USA \\ ${ }^{3}$ Institute for Solid State Physics, the University of Tokyo, \\ 5-1-5 Kashiwanoha, Kashiwa-shi, Chiba 27\%-8581, Japan \\ ${ }^{4}$ Kavli Institute for Theoretical Physics, University of California, Santa Barbara, CA 93106, USA
}

(Dated: February 4, 2020)

\begin{abstract}
We discuss quantum Hall effects in a gapped insulator on a periodic two-dimensional lattice. We derive a universal relation among the the quantized Hall conductivity, and charge and flux densities per physical unit cell. This follows from the magnetic translation symmetry and the large gauge invariance, and holds for a very general class of interacting many-body systems. It can be understood as a combination of Laughlin's gauge invariance argument and Lieb-Schultz-Mattis-type theorem. A variety of complementary arguments, based on a cut-and-glue procedure, the manybody electric polarization, and a fractionalization algebra of magnetic translation symmetry, are given. Our universal relation is applied to several examples to show nontrivial constraints. In particular, a gapped ground state at a fractional charge filling per physical unit cell must have either a nonvanishing Hall conductivity or anyon excitations, excluding a trivial Mott insulator.
\end{abstract}

PACS numbers:

Keywords: Quantum Hall effects, Lieb-Schultz-Mattis theorem, Many-body polarization, Symmetry fractionalization

\section{Contents}

\section{Introduction}

\section{Setup and Main Results}

III. Flux insertion

A. Large gauge invariance and momentum counting

B. Momentum counting on a torus

C. Flux insertion and effective symmetries

IV. Filling-enforced constraint on $\sigma_{x y}$ : a "cut and glue" proof using edge states

V. Many-Body Electric Polarization

A. Defining the many-body polarization as a bulk quantity

B. Many-body polarization in degenerate ground states

VI. Integer Quantum Hall Effect

A. Quantization of Hall conductance

B. IQHE in a periodic system

C. Fluxon stitching picture

VII. Fractional Quantum Hall effects

A. Fractional quantization of the Hall conductivity

B. FQHE in a periodic system

C. Fluxon stitching and braiding with background anyons

VIII. Fractionalization algebra of magnetic translation symmetry
IX. Generalization to non-symmorphic lattices

X. Applications

A. IQHE models

B. Magnetically ordered systems

C. Fractional Quantum Hall States on lattice

XI. Conclusions and Discussions

Acknowledgments

A. Charge pumping and generalized

Luttinger's theorem for edge states of Abelian QHE

\section{B. Berry phase of fluxon braiding in Abelian} QHE

C. Magnetically translating a unit fluxon

References

\section{INTRODUCTION}

12

12

12

13
Quantum Hall effects (QHE) of 2-dimensional electron gas (2DEG) [1, 2] exemplify the first class of topological phases [3], characterized by their quantized bulk response functions and gapless edge excitations. In fact, QHE has been a source of many concepts that have become essential in more general quantum many-body problems. One of the important directions in quantum many-body theory is to find a general constraint based on symme14 tries of the system. Such a constraint would be a guiding 
principle in classifying a wide variety of systems, and sometimes in designing systems with a desired property. QHE has been also instrumental in developing this type of approach. Laughlin's gauge argument for the quantization of the Hall conductance is a beautiful example, which inspired many other developments.

The Hall conductivity may also be constrained by other symmetries. This is evident in the Hall effect of 2DEG in a free 2-dimensional space, where the Hall conductivity $\sigma_{x y}$ is fully determined by filling factor $\nu$, the ratio of electron number density $\bar{\rho}$ and flux density $B / \Phi_{0}$ :

$$
\sigma_{x y}^{2 \mathrm{DEG}}=\nu \frac{e^{2}}{h}, \quad \nu=\frac{\bar{\rho}}{B / \Phi_{0}}=\frac{h \bar{\rho}}{e B} .
$$

Guaranteed by Galilean invariance, this powerful relation remains valid in the presence of interactions, applying to both integer and fractional QHEs in the free space.

QHE on a periodic lattice is a more difficult problem. Despite the complicated nature of the spectrum[4], the Hall conductance is topologically quantized [5] 8$]$. This observation led to the foundation of topological quantum many-body physics, and has also received renewed interest recently [9 12]. The absence of the Galilean invariance in the lattice implies the breakdown of the simple relation (1). Nevertheless, the periodic lattice has a discrete translation symmetry. Therefore, we can ask a natural question: is there a relation similar to (1) for QHEs in a periodic crystal? More specifically, given the electron density and magnetic field, to what extent can we determine the Hall conductivity for QHEs on a discrete lattice? In fact, the Lieb-Schultz-Mattis (LSM) theorem [13] and its generalizations [14-16], which are a filling-enforced constraint [17] on quantum many-body systems, may be also be understood as a remnant of Galilean invariance on a discrete lattice. Therefore it would be natural to expect a generalization of Eq. (1) to lattice systems.

In this paper, we demonstrate that this is indeed the case by deriving a universal relation between the quantized Hall conductivity and filling (particle number per unit cell), which generalizes Eq. (1). In quantum mechanics, a magnetic field must be represented in terms of vector potential. As a consequence, the original, physical translation symmetry is lost in the Hamiltonian in the presence of the magnetic field. This is the case even in the free space. Nevertheless, there is a remnant of the translation symmetry that is called a magnetic translation symmetry [18, 19. Laughlin's gauge argument and (generalized) LSM theorem are both consequences of large gauge invariance. The former constrains the Hall conductivity based on just the gauge invariance and the energy spectrum of the system. The latter constrains the energy spectrum of the system based on the "filling" or the particle density, in the presence of the discrete lattice translation symmetry. Our result is a combination of these two fundamental constraints in quantum manybody problem, relating the quantized Hall conductivity and the particle density. We will give the universal rela- tion 25 first for the integer QHE case, and then later the corresponding result (26) for the fractional QHE. As is the case with the Laughlin's argument and the LSM theorem, our argument is very general and applies to a wide range of interacting systems of either bosons or fermions. While some formulae corresponding to a part of our results were reported earlier [20 22 , our perspective based on the LSM-type theorems elucidates the deep physical meaning of the universal relations. In fact, we will present several applications which demonstrate their surprising power.

The article is organized as follows. We set up the problem and summarize main results in Section [II In Section III. as a preparation, the flux insertion process and the associated momentum counting argument are introduced. Then we prove the filling-enforced constraint on the quantized Hall conductivity for integer QHEs with a "cut and glue" argument, based on gapless edge states in Section IV] In Section V] we review the many-body polarization as formulated by Resta and Sorella. Then the many-body polarization is applied to derive the fillingenforced constraint for IQHEs without relying on the "cut and glue" procedure, in Section VI. The filling-enforced constraint on the quantized Hall conductivity is then generalized for FQHEs in Section VII. An alternative perspective based on a fractionalization algebra of magnetic translation symmetry is discussed in Section VIII In Section IX generalization of our results to non-symmorphic lattices is discussed. Applications of our filling-enforced constraints to various systems of interest are discussed in Section $\mathrm{X}$ before the concluding remarks in Section $\mathrm{XI}$

\section{SETUP AND MAIN RESULTS}

We consider a quantum many-particle system on a periodic two-dimensional potential/lattice in a background magnetic field, with the magnetic flux

$$
\phi=\frac{p}{q},
$$

in the unit of flux quantum $\Phi_{0}=h / e$ per unit cell, where $p$ and $q$ are mutually prime integers. In the following, we set $\hbar=e=1$ so that $\Phi_{0}=2 \pi$. We denote the primitive Bravais vectors of the lattice as $\vec{a}_{1,2}$, and corresponding translation operators as $T_{1,2}$. The continuum version of the Hamiltonian reads

$$
\begin{aligned}
\mathcal{H} & =\int d \vec{r} \frac{1}{2 m} \psi^{\dagger}(\vec{r})\left[(-\mathrm{i} \vec{\nabla}-\vec{A}(\vec{r}))^{2}+V(\vec{r})\right] \psi(\vec{r}) \\
& +\int d \vec{r} \int d \overrightarrow{r^{\prime}} U\left(\vec{r}, \overrightarrow{r^{\prime}}\right) \rho(\vec{r}) \rho\left(\overrightarrow{r^{\prime}}\right),
\end{aligned}
$$

where $\rho \equiv \psi^{\dagger} \psi$ is the particle number density, and $V$ and $U$ are invariant under $T_{1,2}$. It is also convenient to introduce the reciprocal vectors $\vec{g}_{1,2}$ which satisfy

$$
\vec{a}_{\alpha} \cdot \vec{g}_{\beta}=\delta_{\alpha \beta} .
$$


While there is a degree of freedom in gauge choice, in this paper we choose the Landau gauge

$$
\vec{A}(\vec{r})=2 \pi \phi r_{1} \vec{g}_{2},
$$

where

$$
r_{\alpha}=\vec{g}_{\alpha} \cdot \vec{r}
$$

for $\alpha=1,2$. Because of the position dependence of the vector potential, the Hamiltonian is not invariant under the translation $T_{1}$. Nevertheless, one can define a set of magnetic translation operators $\tilde{T}_{1,2}$, which supplement the primitive translations by appropriate gauge transformations and leave the Hamiltonian invariant [18, 19]. For the Landau gauge (5), we choose

$$
\begin{aligned}
& \tilde{T}_{1}=\exp \left(2 \pi \mathrm{i} \phi \int d \vec{r} r_{2} \rho(\vec{r})\right) T_{1}, \\
& \tilde{T}_{2}=T_{2} .
\end{aligned}
$$

These magnetic translation operators satisfy the commutation relation

$$
\tilde{T}_{1} \tilde{T}_{2}=e^{2 \pi \mathrm{i} \phi \hat{N}} \tilde{T}_{2} \tilde{T}_{1}
$$

where

$$
\hat{N}=\int d \vec{r} \hat{\rho}(\vec{r})
$$

is the total charge (in unit of the elementary charge $e$ ) of the system. This "magnetic translation algebra" may be regarded as a defining feature of the system in the presence of the magnetic field. The magnetic translation algebra can be also defined for a lattice model under a uniform magnetic field. Our analysis in the following also applies to such lattice models as well.

In particular, it is helpful to consider a system on the square lattice with a uniform magnetic field, as a simplest example, for the sake of illustrating the problem. For the square lattice with only the nearest-neighbor hoppings, the vector potential on the lattice in the Landau gauge reads

$$
\begin{aligned}
& A_{1}(\vec{r})=0, \\
& A_{2}(\vec{r})=2 \pi \phi r_{1},
\end{aligned}
$$

where $\vec{r}=\left(r_{1}, r_{2}\right)^{T} \in \mathbb{Z}^{2}$ refers to the location of the lattice site. The vector potential enters the hopping term in the Hamiltonian as

$$
\begin{aligned}
& -t_{\vec{r}+\vec{e}_{1}, \vec{r}} e^{\mathrm{i} A_{1}(\vec{r})} c^{\dagger}\left(\vec{r}+\vec{e}_{1}\right) c(\vec{r}) \\
& -t_{\vec{r}+\vec{e}_{2}, \vec{r}} e^{\mathrm{i} A_{2}(\vec{r})} c^{\dagger}\left(\vec{r}+\vec{e}_{2}\right) c(\vec{r})+\text { H.c. }
\end{aligned}
$$

where $\vec{e}_{1}=(1,0)^{T}$ and $\vec{e}_{2}=(0,1)^{T}$. The primitive translations $T_{1,2}$ are just the lattice translations by $\vec{e}_{1,2}$. The magnetic translation operators on the square lattice are then

$$
\begin{aligned}
& \tilde{T}_{1}=\exp \left(2 \pi \mathrm{i} \phi \sum_{\vec{r} \in \mathbb{Z}^{2}} r_{2} n(\vec{r})\right) T_{1}, \\
& \tilde{T}_{2}=T_{2}
\end{aligned}
$$

where $n(\vec{r})$ is the particle number operator at the site $\vec{r}$. They satisfy the same magnetic translaton algebra $(9)$.

The non-commutativity of magnetic translation operators $\tilde{T}_{1}$ and $\tilde{T}_{2}$ prevents us from using the common techniques such as Fourier transforming to the momentum space. Thus it is often convenient to use the commuting set of magnetic translation operators, say $\left(\tilde{T}_{1}\right)^{q}$ and $\tilde{T}_{2}$. This corresponds to considering a "magnetic unit cell", which is $q$ times larger than the original, physical unit cell. Throughout this paper, "unit cell" (without "magnetic" in its front) refers to the original unit cell and not to the magnetic unit cell.

Now let us consider a system defined on a torus. We consider a system consisting of $L_{1} \times L_{2}$ unit cells, namely $0 \leq r_{\alpha}<L_{\alpha}$, where $r_{\alpha}=\vec{g}_{\alpha} \cdot \vec{r}$. The uniformity of the magnetic field including on the boundaries requires the generalized periodic boundary conditions

$$
\psi(\vec{r})=\left(\tilde{T}_{\alpha}\right)^{L_{\alpha}} \psi(\vec{r})
$$

For the Landau gauge (5), it is explicitly given as

$$
\begin{aligned}
& \psi(\vec{r})=e^{2 \pi i \phi L_{1} r_{2}} \psi\left(\vec{r}+L_{1} \vec{a}_{1}\right), \\
& \psi(\vec{r})=\psi\left(\vec{r}+L_{2} \vec{a}_{2}\right) .
\end{aligned}
$$

The consistency of the relation between $\psi(\overrightarrow{0})$ and $\psi\left(L_{1} \vec{a}_{1}+L_{2} \vec{a}_{2}\right)$, which can be obtained by applying $\left(\tilde{T}_{1}\right)^{L_{1}}$ and $\left(\tilde{T}_{2}\right)^{L_{2}}$ in two different orders, requires

$$
\phi L_{1} L_{2} \in \mathbb{Z}
$$

This is equivalent to the condition that the total magnetic flux piercing the system is an integral multiple of the flux quantum $\Phi_{0}$. Therefore, $L_{1} L_{2}$ must be an integral multiple of $q$. Likewise, in a closed system, the total particle number

$$
N=\bar{\rho} L_{1} L_{2}
$$

where $\bar{\rho}$ is the average number of particles per unit cell, must be an integer. If

$$
\bar{\rho}=\frac{p^{\prime}}{q^{\prime}},
$$

where $p^{\prime}$ and $q^{\prime}$ are mutually coprimes, $L_{1} L_{2}$ must contain the factor $q^{\prime}$ as well as $q$.

At this point, there is a freedom in assigning the factor $q$ to $L_{1}$ and $L_{2}$. It is possible to choose $L_{1}$ as an integral multiple of $q$ and $L_{2}$ as a coprime with $q$, or vice versa. However, the choice of $L_{1}$ and $L_{2}$ is further restricted by the requirement of (magnetic) translation symmetry.

In the Landau gauge (5), the magnetic translation operator $\tilde{T}_{2}$ in the 2 -direction is identical to the original lattice translation operator $T_{2}$, as in Eq. (8). It thus appears that the system is always invariant under $\tilde{T}_{2}=T_{2}$. However, for a generic choice of $L_{1}$, the generalized periodic boundary condition (16) required for the torus breaks 
the translation invariance under $T_{2}$. For the system on the torus to be invariant under $\tilde{T}_{2}=T_{2}$, the phase factor $e^{2 \pi i \phi L_{1} r_{2}}$ must be invariant under $r_{2} \rightarrow r_{2}+1$ This requires $\phi L_{1} \in \mathbb{Z}$, namely

$$
L_{1}=q l_{1}
$$

where $l_{1}$ is an integer.

On the other hand, the magnetic translation operator $\tilde{T}_{1}$, Eq. (7), in the present Landau gauge can be written as

$$
\tilde{T}_{1}=\left(\mathcal{U}_{2}\right)^{\phi L_{2}} T_{1}
$$

where

$$
\mathcal{U}_{\alpha}=\exp \left(\frac{2 \pi \mathrm{i}}{L_{\alpha}} \int d \vec{r} r_{\alpha} \hat{\rho}(\vec{r})\right)
$$

for $\alpha=1,2$. On the torus with the (generalized) boundary condition, $\mathcal{U}_{2}$ is nothing but the "fundamental" large gauge transformation along the 2-direction. An integer power of $\mathcal{U}_{2}$ is also a large gauge transformation on the torus, as well. However, a fractional power of $\mathcal{U}_{2}$ is not a well-defined large gauge transformation on the torus. The Hamiltonian on the torus is invariant under the magnetic translation $\tilde{T}_{1}$ only if $\left(\mathcal{U}_{2}\right)^{\phi L_{2}}$ is a large gauge transformation, namely only if

$$
\phi L_{2} \in \mathbb{Z}
$$

This requires $L_{2}$ to be an integral multiple of $q$. In this sense, with the Landau gauge (5), the Hamiltonian on the torus has the magnetic translation invariance under $\tilde{T}_{1}$, only when $L_{2}$ is an integral multiple of $q$. We note that, even when $L_{2}$ is not an integral multiple of $q$, the system on the torus of the size $L_{1} \times L_{2}$ is also perfectly welldefined with uniform flux with the generalized periodic boundary conditions (16) and (17).

The major results of this paper are summarized in the following theorems.

\section{Theorem 1. Filling-enforced constraint on $\sigma_{x y}$ for IQHE:}

In a generic two-dimensional (2d) system of bosons and/or fermions preserving magnetic translation symmetry (9) and $U(1)$ charge conservation, if it has a unique gapped ground state on torus, its Hall conductivity must satisfy the following condition:

$$
\tilde{\sigma}_{x y} \cdot \phi=\bar{\rho} \bmod 1,
$$

where $\tilde{\sigma}_{x y}=\sigma_{x y} \cdot h / e^{2}$ is the Hall conductivity in the unit of $e^{2} / h$, and $\bar{\rho}$ is the number of particles (or the charge in unit of fundamental charge e) per unit cell.

This theorem is the lattice analog of (1) for 2DEG in continuum.

Furthermore, it can be extended to Fractional Quantum Hall effects (FQHEs) with topologically degenerate ground states, as follows:
Theorem 2. Filling-enforced constraint on $\sigma_{x y}$ for FQHE:

In a generic gapped two-dimensional (2d) system of bosons and/or fermions preserving magnetic translation symmetry (9) and $U(1)$ charge conservation, its Hall conductivity $\tilde{\sigma}_{x y}$ in unit of $e^{2} / h$ must satisfy the following condition

$$
\bar{\rho}=\tilde{\sigma}_{x y} \cdot \phi+\frac{\theta_{F, a}}{2 \pi} \bmod 1 .
$$

where $\theta_{F, a}$ is mutual statistical angle between fluxon $(F)$ and background anyon (a) [23, 24] in each unit cell. When there are $n$ degenerate ground states,

$$
n \frac{\theta_{F, a}}{2 \pi} \in \mathbb{Z} .
$$

The non-interacting fermion version of Theorem 11 is known as the Diophantine equation [20]. It was generalized to many-body systems by Avron and Yaffe [21] and studied further in Ref. [22]. The many-body version of the Diophantine equation in Ref. 21] corresponds to the above theorems at a fixed system size and number of particles, with a given number of degenerate ground states. However, we believe that the theorem stated as above in terms of $\phi$ and $\bar{\rho}$ is useful in many ways. For example, it reveals a deep connection to the LSM-type theorems, leading to natural generalizations such as Theorem 3 for non-symmorphic lattices in Section IX. Furthermore, it gives rise to powerful applications, in particular the one showing the stability of gapless phases in $\pi$-flux systems, as we will discuss in Section $\mathrm{X}$. The relation to the statistical angle $\theta_{F, a}$ is also a novel perspective introduced in this paper.

Clearly, Theorem 1 is simply a special case without bulk anyons, of the latter more general Theorem 2. When $\phi=0$ magnetic translation (9) reduces to usual lattice translations, and 26 reduces to the well-known fact that a gapped $2 \mathrm{~d}$ phase with fractional filling per unit cell necessarily leads to topological order if translations $T_{1,2}$ are preserved [16, 25, 27]. As will be shown later, because the magnetic translation symmetry determines only the fractional part of $\phi$, the background anyon $a$ per unit cell has the following ambiguity: When we increase the flux density $\phi$ by $\Delta \phi=1$, there is one extra "anti-fluxon" per unit cell:

$$
\phi \rightarrow \phi+1, \quad a \rightarrow a-F
$$

It then follows that $\theta_{F . a} \rightarrow \theta_{F, a}-\theta_{F, F}$ under this transformation. The consistency of the Theorem 26) with this transformation leads to the identity

$$
e^{\mathrm{i} \theta_{F, F}}=e^{2 \pi \mathrm{i} \tilde{\sigma}_{x y}} .
$$

This is actuall a well known result [27] that Hall conductivity equals the mutual statistical angle of two fluxons in unit of $2 \pi$. 
The theorem 26 can be proved for both Abelian and non-Abelian topological orders, under the assumption of symmetry fractionalization (i.e. we are limited to the situations that magnetic translation symmetry does not change anyon types). Note that even in non-Abelian cases, background anyon a per unit cell must be an Abelian anyon to be compatible to translational symmetry operation in topological orders [24, 27]. Meanwhile, the fluxon must also obey Abelian statistics even in nonAbelian FQH states [27.

\section{FLUX INSERTION}

\section{A. Large gauge invariance and momentum counting}

Suppose that the system is in a ground state initially at $t=0$. Let us define the "flux insertion operators" $\mathcal{F}_{\alpha}(\Phi)$, where $\alpha=1,2$, in the following way. They represent the time evolution operators for a process of adiabatic insertion of flux $\Phi$ through the "hole" encircled by a loop in $\alpha$ direction, where the periodic boundary condition is imposed. It should be noted that, this flux is an Aharonov-Bohm flux which does not directly touch the particles on the two-dimensional system, and should be distinguished from the magnetic flux $\left(\phi \Phi_{0}\right.$ per unit cell) which is piercing through the system (and "perpendicular" to the two-dimensional plane).

For example, we choose the time evolution of the Hamiltonian as

$$
\mathcal{H}_{\alpha}(\Phi, t)=\mathcal{H}_{\alpha}\left(A_{\alpha}=\frac{\Phi}{L_{\alpha}} \frac{t}{T}\right),
$$

for a large enough time $T$. Then

$$
\mathcal{F}_{\alpha}(\Phi) \equiv \mathcal{T} \exp \left(-i \int_{0}^{T} d t \mathcal{H}_{\alpha}(\Phi, t)\right)
$$

where $\mathcal{T}$ denotes the time ordering. After the flux insertion process $\mathcal{F}_{\alpha}(\Phi)$, the Hamiltonian becomes $\mathcal{H}(\Phi)$ which contains the flux $\Phi$.

On the other hand, the large gauge transformation is characterized by the identity

$$
\mathcal{H}_{\alpha}\left(\Phi_{0}\right)=\mathcal{U}_{\alpha} \mathcal{H}_{\alpha}(0)\left(\mathcal{U}_{\alpha}\right)^{-1}
$$

Thus, if we consider the insertion of unit flux quantum $\Phi_{0}$ by $\mathcal{F}_{\alpha}\left(\Phi_{0}\right)$, the inserted flux can be eliminated precisely by the large gauge transformation 23. It is thus convenient to consider the combined operation

$$
\tilde{\mathcal{F}}_{\alpha}\left(\Phi_{0}\right) \equiv\left(\mathcal{U}_{\alpha}\right)^{-1} \mathcal{F}_{\alpha}\left(\Phi_{0}\right),
$$

of the insertion of the unit flux quantum $\Phi_{0}$ and the large gauge transformation. This is the operator what has been used in various contexts [15, 28, 29. After this process, we get back to the original Hamiltonian $\mathcal{H}_{\alpha}(0)$.
However, in general, the final state $\tilde{\mathcal{F}}_{\alpha}\left(\Phi_{0}\right)\left|\Psi_{I}\right\rangle$ after the evolution is different from the initial state $\left|\Psi_{I}\right\rangle$.

To see this, it is useful to invoke the "momentum counting" argument [13 15]. As in Sec. II] let us consider the system consists of $L_{1} \times L_{2}$ unit cells. In general, we have

$$
T_{\alpha} \mathcal{U}_{\alpha} T_{\alpha}^{-1} \mathcal{U}_{\alpha}^{-1}=e^{-\frac{2 \pi \mathrm{i}}{L_{\alpha}} \int d \vec{r} \rho(\vec{r})}=e^{-2 \pi \mathrm{i} \bar{\rho} L_{\bar{\alpha}}},
$$

where $\overline{1}=2$ and $\overline{2}=1$. In our setup with the Landau gauge (5), $\tilde{T}_{2}=T_{2}$ commutes with the Hamiltonian, when the periodic boundary condition is imposed in 2direction. Thus the total momentum $P_{2}$ in 2-direction defined by

$$
T_{2}=e^{i P_{2}}
$$

is a good quantum number, modulo $2 \pi$.

On the other hand, since the introduction of the uniform vector potential corresponding to flux insertion in the hole does not break the translation symmetry,

$$
\left[T_{2}, \mathcal{F}_{2}(\Phi)\right]=0
$$

Combining this and Eq. (34) for $\alpha=2$ with the definition 33 , we find

$$
T_{2} \tilde{\mathcal{F}}_{2}\left(\Phi_{0}\right)=\tilde{\mathcal{F}}_{2}\left(\Phi_{0}\right) T_{2} e^{2 \pi \mathrm{i} \bar{\rho} L_{1}} .
$$

Eq. (37) implies that the momentum $P_{2}$ of the final state differs from that of the initial state by

$$
\Delta P_{2} \equiv 2 \pi \bar{\rho} L_{1} \quad \bmod 2 \pi \text {. }
$$

If $\bar{\rho} L_{1}$ is not an integer, the final state $\tilde{\mathcal{F}}_{\alpha}\left(\Phi_{0}\right)\left|\Psi_{I}\right\rangle$ must be orthogonal to the initial state $\left|\Psi_{I}\right\rangle$.

We note that, up to this point, we do not need an assumption of adiabaticity of the flux insertion process. The present result is valid however fast the flux is inserted, and whether the system has an excitation gap or not. Moreover, we assumed the periodic boundary condition in the 2-direction, but not in the 1-direction at this point. Thus the momentum counting (38) is also valid when the open boundary condition is imposed in the 1-direction, for example.

In the application to QHE, a system with open boundaries generally has gapless edge states, even when there is a non-vanishing excitation gap in the bulk. The flux insertion/momentum counting argument can still be useful in the presence of the gapless edge states, as we will discuss later in Section IV.

\section{B. Momentum counting on a torus}

In fact, it is often convenient to consider gapped system without edge states. For this, we impose the periodic boundary conditions in both directions, or equivalently consider the system on a torus of the size $L_{1} \times L_{2}$. This introduces several subtleties. First, to make the system well-defined on the torus, the total magnetic flux piercing 
the system must be an integral multiple of the unit flux quantum, as in Eq. (18). It follows that $L_{1} L_{2}$ must be an integral multiple of $q$.

As we have discussed in Section II] there is some freedom in choosing $L_{1}$ and $L_{2}$. Since the translation invariance under $T_{2}$ is essential for the momentum counting argument, on the torus we have to choose $L_{1}$ to be an integral multiple of $q$, as in Eq. (21). Under this condition, Eq. (34) for $\alpha=2$ reads

$$
T_{2} \mathcal{U}_{2} T_{2}{ }^{-1} \mathcal{U}_{2}^{-1}=e^{-\frac{2 \pi \mathrm{i}}{L_{2}} \int d \vec{r} \rho(\vec{r})}=e^{-2 \pi \mathrm{i} \bar{\rho} q l_{1}},
$$

and thus the momentum gain due to the flux insertion (and large gauge transformation) $\tilde{\mathcal{F}}_{2}\left(\Phi_{0}\right)$ is

$$
\Delta P_{2} \equiv 2 \pi \bar{\rho} L_{1} \quad \bmod 2 \pi \equiv 2 \pi \bar{\rho} q l_{1} \quad \bmod 2 \pi .
$$

$l_{1}$ can be any integer, and we choose it as a coprime with $q^{\prime}$.

In the presence of the gap, we may require flux insertion process $\mathcal{F}$ to be adiabatic, assuming that the gap never closes during the flux insertion [15]. Under this condition, if the initial state $\left|\Psi_{I}\right\rangle$ is taken as a ground state $\left|\Psi_{0}\right\rangle$, which is also an eigenstate of $T_{2}$, the final state $\tilde{\mathcal{F}}_{2}\left(\Phi_{0}\right)\left|\Psi_{0}\right\rangle$ must also be a ground state. Then, if $\bar{\rho} q$ is not an integer, there must be multiple degenerate ground states, or gapless excitations. This is the generalized LSM theorem applied to a system under uniform magnetic field $(\phi=p / q$ flux per unit cell). The statements of the standard LSM theorem apply, by replacing $\bar{\rho}$ by $\bar{\rho} q$. Namely, under the magnetic field, the particle number per magnetic unit cell, which is $q$ times larger than the original unit cell, is the relevant parameter for the generalized LSM theorem. In particular, if we assume a unique ground state with a gap on the torus,

$$
\bar{\rho} q \in \mathbb{Z} \Leftrightarrow \bar{\rho} \phi=0 \bmod 1,
$$

or equivalently $q / q^{\prime} \in \mathbb{Z}$. This corresponds to the integer number of particles per magnetic unit cell. In the limit of non-interacting electrons, Eq. (41) allows the Fermi level to lie in a band gap between bands defined on the magnetic Brillouin zone. However Eq. (41) holds in more general interacting systems, as long as the system is gapped with the unique ground state on a torus.

At this point, the LSM theorem gives no constraint when $\bar{\rho} q \in \mathbb{Z}$, even if $\bar{\rho}$ is fractional. Nevertheless, in the following, we will go beyond the LSM theorem and show that a different type of general constraint arises even when $\bar{\rho} q \in \mathbb{Z}$.

\section{Flux insertion and effective symmetries}

We have seen that the adiabatic insertion of the unit flux quantum $\mathcal{F}_{\alpha}\left(\Phi_{0}\right)$ and the fundamental large gauge transformation $\mathcal{U}_{\alpha}$ have a similar effect of introducing the unit flux quantum $\Phi_{0}$ in the "hole" of the torus. However, they are quite different operators (and thus $\tilde{\mathcal{F}}_{\alpha}\left(\Phi_{0}\right) \neq 1$ ).
In fact, $\mathcal{F}_{\alpha}$ correspond to application of an electric field in $\alpha$-direction, while $\mathcal{U}_{\alpha}$ does not. Physically, the applied electric field can accelerate particles. Thus, the flux insertion process generally changes the energy of a given initial state. As a consequence, the analog of Eq. (32), generally does not hold for $\mathcal{F}_{\alpha}(\Phi)$, even for $\Phi=\Phi_{0}$. Nevertheless, when the system is gapped, the adiabaticity of $\mathcal{F}_{\alpha}$ implies that

$$
\mathcal{H}_{\alpha}\left(\Phi_{0}\right) \sim \mathcal{F}_{\alpha}\left(\Phi_{0}\right) \mathcal{H}_{\alpha}(0)\left(\mathcal{F}_{\alpha}\left(\Phi_{0}\right)\right)^{-1}
$$

holds, when restricted onto the ground-state subspace of the entire Hilbert space. (This holds because the initial and final states are both ground state(s) of the physically equivalent Hamiltonian and thus have the same eigenvalue.) It also follows that

$$
\left[\tilde{\mathcal{F}}_{\alpha}\left(\Phi_{0}\right), \mathcal{H}(0)\right] \sim 0
$$

in the ground-state subspace. In this sense, $\tilde{\mathcal{F}}_{\alpha}\left(\Phi_{0}\right)$ effectively acts as a symmetry generator, as far as the ground states are concerned.

Furthermore, one can certainly consider an adiabatic flux insertion, $\mathcal{F}_{\alpha}(\Phi)$, for any $\Phi$ which is not necessarily an integer multiple of the unit flux quantum $\Phi_{0}$. Let us consider a finite-size system of $L_{1} \times L_{2}$ unit cells on a torus with the flux (2) per unit cell, in the Landau gauge (5) (or its lattice version). Let us choose $L_{1}$ to be an integral multiple of $q$, as in Eq. (21), so that the system retains the translation symmetry $T_{2}$,

In order to derive strongest possible restrictions, we choose $L_{2}$ to be a coprime with $q$. As we remarked in Sec. II. this choice is allowed as $L_{1}$ is already chosen to be an integral multiple of $q$. A drawback of this choice of $L_{2}$ is that, the magnetic translation operator $\tilde{T}_{1}$ is in fact not well-defined because $\phi L_{2}$ is not an integer, as we have discussed in Eq. (22). Nevertheless, here we can use the adiabatic flux insertion, instead of the large gauge transformation, to define an analogue of the magnetic translation operator. The adiabatic flux insertion $\mathcal{F}_{2}\left(\phi L_{2} \Phi_{0}\right)$ exactly compensates the change in the vector potential induced by the primitive translation $T_{1}$. Therefore,

$$
\tilde{\tilde{T}}_{1} \equiv \mathcal{F}_{2}\left(\phi L_{2} \Phi_{0}\right) T_{1}
$$

maps a ground state of the original Hamiltonian $\mathcal{H}(0)$ to a ground state of the same Hamiltonian, and thus can be regarded as another "effective symmetry generator" in the same sense as Eq. (43). This may be regarded as a remnant of the magnetic translation symmetry on the torus, although the magnetic translation operator $\tilde{T}_{1}$ in the standard sense is ill-defined in this setup.

As discussed earlier, $\phi L_{2}$ not being an integer does not prevent adiabatically inserting the "fractional flux" $\phi L_{2} \Phi_{0}$, while the large gauge transformation is ill-defined for a fractional flux. From the commutation relations 62 and 34 , we find

$$
\tilde{\tilde{T}}_{1} \mathcal{U}_{1}=\mathcal{U}_{1} \tilde{\tilde{T}}_{1} e^{2 \pi \mathrm{i} L_{2}\left(\phi \tilde{\sigma}_{x y}-\bar{\rho}\right)} .
$$


This operator $\tilde{\tilde{T}}_{1}$ will prove useful, as we will demonstrate later in Section VIB

\section{FILLING-ENFORCED CONSTRAINT ON $\sigma_{x y}:$ A "CUT AND GLUE" PROOF USING EDGE STATES}

In the following we give a heuristic proof of the main theorem 25) for integer QHEs. This is based on a "cut and glue" procedure, by examining how the gapless edge states which appears upon cutting the system, respond to flux insertion. It can be straightforwardly generalized to non-symmorphic lattices, leading to a stronger condition (98).

We first consider the system on a torus of size $L_{1} \times L_{2}$, as discussed in the previous section. To represent the uniform magnetic field, we adopt the Landau gauge (5). Here we choose $L_{1}=q l_{1}, l_{1}=n_{1} q+1$, and $L_{2}=n_{2} q$ with $n_{1,2} \in \mathbb{Z}$. With this choice, we satisfy the requirement (18). Furthermore, the magnetic translation operators $T_{1,2}$ are well-defined and commute with the Hamiltonian, as both conditions (21) and (24) are satisfied.

As illustrated in FIG. 1 this torus can be cut into $q$ cylinders of the size $l_{1} \times L_{2}$, where $L_{2}=n_{2} q$. We choose $l_{1}$ to be mutually prime with $q^{\prime}$ and $n_{2}=0 \bmod q^{\prime}$ so that each cylinder can have an integer total number of elementary particles, while keeping the fractional number $\bar{\rho}=p^{\prime} / q^{\prime}$ of particle per unit cell. While this is not the only possible choice of the system size, it turns out to be convenient as we will see in the following. When $n_{1,2}$ are large enough, each cylinder can be treated as a macroscopic system. We emphasize that, although these $q$ cylinders are physically identical to each other, the corresponding Hamiltonians are not identical. This can be seen by going back to the torus before it was cut into cylinders and recalling the Landau gauge $(5)$ or its lattice version such as Eq. (11). The Hamiltonians for two different cylinders are related by the magnetic translation operator $\left(\tilde{T}_{1}\right)^{l_{1}}$ or its integer powers, not the simple translation.

In this Section, we assume that the system on the torus has a non-vanishing gap and has a unique ground state. This means that here we consider an Integer Quantum Hall state, since a Fractional Quantum Hall state must have topologically degenerate ground states on a torus [30. We will discuss Fractional Quantum Hall states later.

First we adiabatically insert a unit flux quantum $\Phi_{0}$ through each cylinder (red arrow in FIG. 1). Although each cylinder which appears after the cut does not have a translation invariance in the 1-direction, we can still apply the momentum counting argument in the 2-direction as discussed in Section IIIA As a result, for each cylinder, we find that the momentum in the 2-direction acquires the shift (38) where $L_{1}$ is replaced by the width of

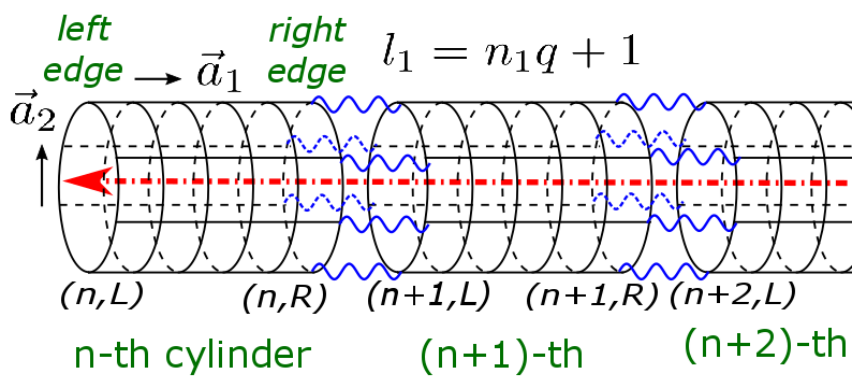

Figure 1: (color online) An illustration of the flux insertion and cut-and-glue procedure. Red arrow of dotted dash line denotes inserting $2 \pi$ flux adiabatically through the torus,

the cylinder $l_{1}$, namely

$$
\Delta P_{2}=2 \pi \bar{\rho} l_{1} .
$$

Because of our choice of $l_{1}$ so that it is a coprime with $q^{\prime}$, this is nontrivial modulo $2 \pi$ and thus the initial state and the final state after the flux insertion $\tilde{\mathcal{F}}\left(\Phi_{0}\right)$ must be orthogonal. Following the logic of Refs. [13, 15, this leaves us two possibilities: (i) there are (at least) two (quasi)degenerate ground states below the gap, or (ii) there are gapless excitations.

Our assumption that the system has a non-vanishing gap with a unique ground state on the torus then implies the presence of gapless edge states on the cylinder. Thus, under the assumption of a unique gapped ground state on a torus, fractional filling with respect to the original, physical unit cell $\bar{\rho} \notin \mathbb{Z}$ necessarily implies gapless edge states in each cylinder. Upon adiabatic insertion of one flux quantum, a momentum shift $(46)$ is acquired via level crossings on the two edges of each cylinder. By denoting the momentum transfer through the left(right) edge of $n$-th cylinder (see FIG. 1) as $\Delta P_{2, L(R)}^{(n)}$, we have

$$
\begin{gathered}
\Delta P_{2, L}^{(n)}+\Delta P_{2, R}^{(n)} \equiv \Delta P_{2}=2 \pi \bar{\rho} l_{1} \quad \bmod 2 \pi, \\
n=1,2, \cdots, q .
\end{gathered}
$$

As illustrated in FIG. 2, the momentum transfer for each edge is always proportional to charge density $\rho_{n, L / R}$ (in unit of $e$ ) on each edge:

$$
\Delta P_{2, L(R)}^{(n)}=2 \pi \rho_{n, L(R)}, \quad \forall n .
$$

This can be viewed as a generalization of Luttinger's theorem to interacting systems. In Appendix A we proved this generalized Luttinger's theorem in the chiral boson description of edge states where the gapped bulk is captured by an Abelian Chern-Simons theory 31 .

On the other hand, the $(n+1)$-th cylinder is related to the $n$-th cylinder by magnetic translation $\left(\tilde{T}_{1}\right)^{l_{1}}$, which is a combination of pure translation $T_{1}^{l_{1}}$ and large gauge transformation $\mathcal{U}_{2}^{\phi l_{1} L_{2}}$.

Therefore the $(n+1)$-th cylinder can be viewed as translating $n$-th cylinder by $l_{1}=n_{1} q+1$ unit cells along 


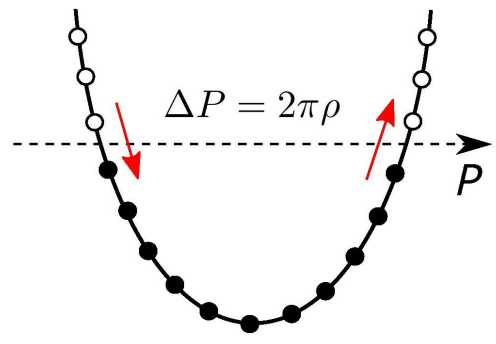

Figure 2: (color online) An intuitive picture for generalized Luttinger's theorem 48.

$\vec{a}_{1}$ direction, and then applying large gauge transformation $\left(\mathcal{U}_{2}\right)^{\phi l_{1} L_{2}}=\left(\mathcal{U}_{2}\right)^{p n_{2} l_{1}}$ flux quanta through it. Due to the well-known chiral anomaly [32] in $1+1-\mathrm{D}$, the chiral edge states of QHEs is not invariant under large gauge transformations 31. As shown in Appendix A a large gauge transformation $\left(\mathcal{U}_{2}\right)^{\phi l_{1} L_{2}}$ can change the charge density of edge states by $\mp \phi l_{1} \sigma_{x y}$ for left(right) edges, where $\sigma_{x y} \in \mathbb{Z}$ is the integer Hall conductance of the bulk. This leads to the following relation on the charge density on the edges:

$$
\begin{aligned}
& \rho_{n+1, L}=\rho_{n, L}-\sigma_{x y} \phi l_{1}, \\
& \rho_{n+1, R}=\rho_{n, R}+\sigma_{x y} \phi l_{1} .
\end{aligned}
$$

Now let's glue the right edge of $n$-th and the left edge of $(n+1)$-th cylinder together preserving translation $T_{2}$, and the entity of these two cylinders becomes a larger cylinder of size $2 l_{1} \times L_{2}$. With a bulk excitation gap, such a gluing procedure won't change the charge density beyond a finite correlation length and hence the charge densities on two edges of the large cylinder are $\rho_{n, L}$ and $\rho_{n+1, R}$. Repeating the flux insertion argument for this large cylinder yields

$$
2 \pi\left(\rho_{n, L}+\rho_{n+1, R}\right)=2 \Delta P_{2}=4 \pi \bar{\rho} l_{1} \bmod 2 \pi .
$$

Combining (50) with 47)- 49) we obtain

$$
2 \pi \sigma_{x y} \phi l_{1}=2 \pi \bar{\rho} l_{1} \quad \bmod 2 \pi
$$

A unique gapped ground state implies an integer $\sigma_{x y} \in \mathbb{Z}$ without fractionally charged quasiparticles. By choosing $l_{1}=n_{1} q+1$ and $n_{1}=0 \bmod q^{\prime}$ for rational flux $\phi=p / q$ and commensurate filling $\bar{\rho}=p^{\prime} / q^{\prime}$, clearly this leads to theorem 25.

\section{MANY-BODY ELECTRIC POLARIZATION}

\section{A. Defining the many-body polarization as a bulk quantity}

The argument in the previous section suggests the universal constraint based on the transport of electrons between the edge states. However, there is a subtlety in the "cut and glue" procedure, namely in the relation between the torus without cuts and the cylinders obtained with the cuts. In addition, the existence of the gapless edge state makes the argument somewhat tricky. However, when the system is gapless, the flux insertion process cannot be adiabatic in the strict sense. Although the momentum counting part of the argument does not actually depend on the adiabaticity, it is difficult to control the final state in the absence of the gap.

In fact, the same problem exists in the celebrated Laughlin's gauge invariance argument for the quantization of Hall conductance, as it depends on a cylinder geometry and accompanying gapless edge states. While it may be still possible to define an adiabatic process in a finite-size system, where generically there is a nonvanishing gap, it is theoretically desirable to invoke an adiabatic argument for a gapped system. For this, it is advantageous to close the argument entirely within the system with periodic boundary conditions, namely on a torus. With the periodic boundary conditions, there is no gapless edge states. The lack of edge states then appears to imply that we cannot define the charge transport. Fortunately, this is not quite the case. In fact, there have been developments over several decades on how to define the electric polarization with periodic boundary conditions. This led to a beautiful formulation of electric polarization of free electron systems in terms of geometric phases. The theory is further generalized to interacting many-body systems. What we would like to point out here is that the theory of polarization in many-body systems is closely related to large gauge invariance, and that we can derive useful identities based on the relation.

Polarization vector $\vec{P}$ is roughly defined as electric dipole moment per unit volume. On average, thus,

$$
\overline{\vec{P}} \sim \frac{1}{V}\left(\int d \vec{r} \vec{r} \rho(\vec{r})\right)+\vec{P}_{0},
$$

where $\vec{P}_{0}$ is the contribution from the immobile background ("ions") and $V$ is the volume of the system. If the system is periodic with Bravais vectors $\vec{a}_{\alpha}$, the bulk of the system is invariant under the translation $\vec{r} \rightarrow \vec{r}+\vec{a}_{\alpha}$ $(\alpha=1,2)$. With the naive definition of the polarization (51), however, it is not invariant under the translation. This means that the polarization vector has some ambiguity in the presence of periodic boundary conditions. On the other hand, the change of $\vec{P}$ during a given process should be uniquely defined. In fact,

$$
\vec{j}=\frac{\partial \vec{P}}{\partial t}
$$

is the current density, which is a well-defined physical quantity even with the periodic boundary conditions. This suggests that it is possible to study polarization even with the periodic boundary conditions. In fact this has been a subject of intense studies over several decades 33, 34. Here we summarize the theory of polarization in many-body interacting systems with periodic 
boundary conditions developed by Resta and Sorella [35. Furthermore, we point out its relation to the large gauge invariance, and discuss the consequences.

As in the previous Section, let us consider a system consisting of $L_{1} \times L_{2}$ unit cells on a torus. We define the "total polarization" in $\alpha$-direction as

$$
\mathcal{P}_{\alpha} \equiv \frac{1}{L_{\alpha}} \cdot\left(\int d \vec{r} \cdot r_{\alpha} \rho(\vec{r})\right),
$$

where $r_{\alpha}$ is defined in Eq. (6). Comparing with Eq. (51), $\overline{\vec{P}}$ and $\overrightarrow{\mathcal{P}}$ are related as

$$
\mathcal{P}_{\alpha}=L_{\bar{\alpha}} \vec{g}_{\alpha} \cdot \overline{\vec{P}}+\text { const., }
$$

for $\alpha=1,2$. This is naturally related to the total current flowing in each direction:

$$
I_{\alpha}=e \frac{\partial \mathcal{P}_{\alpha}}{\partial t} .
$$

One can easily see that $\mathcal{P}_{1}$ is invariant under $T_{2}$. On the other hand, under $T_{1}$, we find the change in

$$
\Delta \mathcal{P}_{1}=\left[T_{1}, \mathcal{P}_{1}\right]=\frac{1}{L_{1}} \int d \vec{r} \vec{g}_{1} \cdot \vec{a}_{1} \rho(\vec{r})=\frac{N}{L_{1}}=\bar{\rho} L_{2},
$$

where $\vec{r}$ refers to the lattice sites and $n_{\vec{r}}$ is the particle number operator at the site $\vec{r}$. Therefore, the total polarization $\mathcal{P}_{1}$ would be only well-defined modulo $\bar{\rho} L_{2}=p^{\prime} L_{2} / q^{\prime}$.

Resta and Sorella [35] discussed the electric polarization in general interacting many-body systems. They introduced an exponential of the total polarization operator $(53)$ as

$$
\mathcal{U}_{\alpha}=e^{2 \pi i \mathcal{P}_{\alpha}}
$$

They considered its ground-state expectation value

$$
z_{\alpha} \equiv\left\langle\mathcal{U}_{\alpha}\right\rangle,
$$

and then argued that the total polarization $\overline{\mathcal{P}}_{\alpha}$ of the ground state can be defined as

$$
z_{\alpha}=\left|z_{\alpha}\right| e^{2 \pi i \overline{\mathcal{P}}_{\alpha}} .
$$

This would determine $\overline{\mathcal{P}}_{\alpha}$ modulo integer. $\overline{\mathcal{P}}_{\alpha}$ would be ill-defined if the expectation value $z_{\alpha}$ vanishes. Resta and Sorella argued that the vanishing of $z_{\alpha}$ (in the thermodynamic limit) implies that the system is conductor in which the polarization is ill-defined. According to them, on the other hand, $\left|z_{\alpha}\right|$ should approach 1 in insulators so that the polarization $\mathcal{P}_{\alpha}$ has vanishing fluctuations and takes the definite value $\overline{\mathcal{P}}_{\alpha}$. (See also Refs. [36, 37]) In this paper we assume that this statement holds in insulators including Quantum Hall states.

In fact, the operator (57) is nothing but the fundamental large gauge transformation operator (or equivalently the LSM twist operator)) (23), as pointed out in Ref. [38]. However, so far the large gauge invariance has not been exploited in the context of the Resta-Sorella theory of many-body polarization. The large gauge invariance based on $\mathcal{U}_{\alpha}$ has been quite useful in deriving various universal constraints on quantum many-body problem. It is one of the goals of the present paper to develop new applications of the large gauge invariance in the context of the many-body polarization. In later Sections, we shall demonstrate that it is indeed fruitful.

\section{B. Many-body polarization in degenerate ground states}

In the presence of the ground-state degeneracy, we have to be careful with the Resta-Sorella definition of the polarization. In particular, when the ground-state degeneracy is required by the LSM theorem, the translation operator $T_{\alpha}$ and the large gauge transformation $\mathcal{U}_{\alpha}$ do not commute (see for example Eq. (39)). It immediately follows that, in a translationally invariant ground state (eigenstate of the translation operator), the expectation value (58) vanishes. This is due to a kinematical reason, and does not mean that the system is conductor. Aligia and Ortiz [39] proposed to use the expectation value of $\left(\mathcal{U}_{\alpha}\right)^{n}$, when the ground states are required to be $n$-fold degenerate by the LSM theorem. It has a non-vanishing expectation value in the translationally invariant ground state, from which one can define the polarization modulo $1 / n$. While their proposal is interesting and valid, it would lead to a weaker constraint in our application. Thus we attempt to utilize the expectation value of $\mathcal{U}_{\alpha}$ itself even in the presence of the ground-state degeneracy. A physical way to understand the degeneracy is as follows. The ground-state degeneracy can be attributed to the formation of "charge-density wave" along the $\alpha$ direction. In two (and higher) dimensions, the groundstate degeneracy can be actually a topological degneracy without any local order parameter. However, even in such a case, regarding the system as a one-dimensional system along the $\alpha$-direction, the ground-state degeneracy can be attributed to the charge-density wave formation. This has been known for example in quantum Hall states [40, 41].

The translationally invariant ground states are given by linear superpositions (Fourier transforms) of the "physical" charge-density wave ground states. Each of the physical charge-density wave ground state would have a definite total polarization, and is related to the other charge-density wave ground states by the lattice translation $T_{\alpha}$. This implies that, each of these states has a non-vanishing expectation value $\left\langle\mathcal{U}_{\alpha}\right\rangle$, which determines the total polarization via Eqs. (58) and (59). Furthermore, in each of these states, following Refs. [35]37], we assume that the fluctuation of the total polarization $\mathcal{P}_{\alpha}$ asymptotically vanishes in the thermodynamic limit. 


\section{INTEGER QUANTUM HALL EFFECT}

\section{A. Quantization of Hall conductance}

Now let us discuss a simple application of the manybody polarization and the large gauge invariance. When a magnetic field perpendicular to the $x y$-plane is applied, we expect Hall effect, which is characterized by a nonvanishing Hall conductivity $\sigma_{x y}$. In a quantum system, under certain conditions, $\sigma_{x y}$ is quantized to integral (rational) multiples of $e^{2} / h$. This is the celebrated Integer (Fractional) Quantum Hall Effect.

Laughlin demonstrated 42] that the large gauge invariance leads to the quantization of $\sigma_{x y}$ in IQHE. This argument was later extended to FQHE 30. However, the original argument involves open boundary conditions, which accompany gapless edge states. This complicates theoretical analysis, in particular the use of the adiabatic process. An alternative approach based on generalized periodic boundary condition is later developed by Niu, Thouless, and Wu [7]. While this approach resolves some of the subtleties of the Laughlin's argument, the appealing simplicity and physical intuition of the argument is lost to some extent. Here, we show that, by considering torus (rectangle with periodic boundary conditions) without edge states, Laughlin's gauge invariance argument can be more directly rephrased in a theoretically better controlled way.

Discussion of IQHE/FQHE is also instructive in introducing a few operators which will be useful in later discussion. While we are mostly interested in periodic systems (which are invariant under discrete translations) in this paper, we allow more general systems without any translation symmetry, which may contain impurities (random potentials). We just assume that the total number of particles is conserved, the particles couple to the external (electromagnetic) U(1) gauge field, and the system has a gap above the ground state.

Now we consider the adiabatic insertion of the magnetic flux $\Phi$ in the "hole" of the torus encircled by the curve in 2-direction, over the period of time $T$. This induces the total "voltage drop" in 2-direction (integration of the electric field $E_{2}$ )

$$
\Delta V_{2}=\frac{\Phi}{T}
$$

during the process. As a consequence, an electric current

$$
I_{1}=\sigma_{x y} \Delta V_{2}=\sigma_{x y} \frac{\Phi}{T}
$$

is induced. With Eqs. (55) and (23), we find

$$
\mathcal{F}_{2}(\Phi) \mathcal{U}_{1}=\mathcal{U}_{1} \mathcal{F}_{2}(\Phi) e^{2 \pi \mathrm{i} \frac{\Phi \sigma_{x y}}{e}}
$$

In particular, following Laughlin's original argument, if we choose the inserted flux as $\Phi=\Phi_{0}$ (unit flux quan- tum),

$$
\mathcal{F}_{2}\left(\Phi_{0}\right) \mathcal{U}_{1}=\mathcal{U}_{1} \mathcal{F}_{2}\left(\Phi_{0}\right) e^{2 \pi \mathrm{i} \frac{\Phi_{0} \sigma x y}{e}}=\mathcal{U}_{1} \mathcal{F}_{2}\left(\Phi_{0}\right) e^{2 \pi \mathrm{i} \tilde{\sigma}_{x y}}
$$

On the other hand, the large gauge transformations in two directions obviously commute:

$$
\mathcal{U}_{1} \mathcal{U}_{2}=\mathcal{U}_{2} \mathcal{U}_{1}
$$

It thus follows that

$$
\tilde{\mathcal{F}}_{2}\left(\Phi_{0}\right) \mathcal{U}_{1}=\mathcal{U}_{1} \tilde{\mathcal{F}}_{2}\left(\Phi_{0}\right) e^{2 \pi \mathrm{i} \tilde{\sigma}_{x y}}
$$

As we have discussed, $\tilde{\mathcal{F}}_{y}\left(\Phi_{0}\right)$ maps a ground state to a ground state.

Resta and Sorella argued that, insulators can be characterized by the well-definedness of the electric polarization. Mathematically, an insulator is characterized by a non-vanishing ground-state expectation value $\left\langle\mathcal{U}_{\alpha}\right\rangle$ in the thermodynamic limit, so that the electric polarization can be defined by its argument (modulo the uncertainty). Let us assume that the system is in a Quantum Hall state and thus is an insulator (in the sense that the diagonal conductivity vanishes). Furthermore, we assume that the system has a gap 62 .

Following Resta-Sorella argument, for the unique ground state $\left|\Psi_{0}\right\rangle$,

$$
\left\langle\Psi_{0}\left|\mathcal{U}_{1}\right| \Psi_{0}\right\rangle=z \neq 0
$$

From the preceding analysis, $\tilde{\mathcal{F}}_{2}\left(\Phi_{0}\right)\left|\Psi_{0}\right\rangle \sim\left|\Psi_{0}\right\rangle$ (up to a phase factor), and thus

$$
\left\langle\Psi_{0}\left|\left(\tilde{\mathcal{F}}_{2}\left(\Phi_{0}\right)\right)^{-1} \mathcal{U}_{1} \tilde{\mathcal{F}}_{2}\left(\Phi_{0}\right)\right| \Psi_{0}\right\rangle=z \neq 0 .
$$

Comparing these with Eq. 65, we find $\frac{\Phi_{0}}{e} \sigma_{x y} \in \mathbb{Z}$, namely the quantization of the Hall conductivity

$$
\sigma_{x y}=\frac{e^{2}}{h} \times \text { integer. }
$$

We note that, in this derivation, translation invariance is not used and therefore the result is applicable to general, non-periodic systems.

While this is just an alternative formulation of Laughlin's gauge argument, it is interesting that the same conclusion can be derived for a system on a torus without edge states, using Resta-Sorella many-body polarization operator. As we will show in the following, the present approach also has several non-trivial generalizations.

\section{B. IQHE in a periodic system}

Now let us re-derive the main result 25 for IQHE in a periodic system, based on the many-body polarization. All the arguments in Sec. VIA still apply, and thus we have the quantization of the Hall conductance 68 holds. Moreover, we consider the system on a torus of $L_{1} \times L_{2}$ 
unit cells, with $L_{1}=q l_{1}$ and $L_{2}$ being a coprime with $q$. The system then has the translation symmetry $T_{2}$, and the effective "translation" symmetry $\tilde{\tilde{T}}_{1}$ as defined in Eq. 44.

As in Sec. VI A, we assume that the system is a gapped insulator and has a unique ground state. Then, the ground state has a non-vanishing expectation value of $\mathcal{U}_{1}$, and the ground state is mapped to itself by $\tilde{\tilde{T}}_{1}$. Thus we immediately find that the phase factor in the commutation relation 45 must be unity. This implies

$$
L_{2}\left(\phi \tilde{\sigma}_{x y}-\bar{\rho}\right) \in \mathbb{Z} .
$$

Since this holds for any $L_{2}$ which is coprime with $q$, with Eqs. (2), (20), and (41), we find the filling-enforced constraint on the quantized Hall conductivity for IQHE (Theorem 1).

\section{Fluxon stitching picture}

We can formulate the flux insertion/large gauge transformation in a different, more "local" manner, by inserting a flux tube of unit flux quantum $\Phi_{0}$ orthogonal to the two-dimensional system, and then dragging it across the system [43. After "stitching" the system by dragging the flux tube along a fundamental cycle of the torus, the "hole" of the torus corresponding to the other fundamental cycle acquires the unit flux quantum $\Phi_{0}$. Thus we can expect that this process is topologically equivalent to the adiabatic insertion of the unit flux quantum $\mathcal{F}_{\alpha}$ introduced earlier.

More precisely, this process can be defined as follows. First we define the operator $f_{\alpha}(\vec{r})$ which corresponds to an "adiabatic" increase of vector potential in $\alpha$ direction by $\Phi_{0} / l_{\alpha}$, over the local region of $l_{1} \times l_{2}$ unit cells located at $\vec{r}$. When we apply $f_{\alpha}(\vec{r})$ to a ground state (vacuum) $\left|\Psi_{0}\right\rangle, f_{\alpha}(\vec{r})\left|\Psi_{0}\right\rangle$, a flux tube with the unit flux quantum $\Phi_{0}$ piercing through two-dimensional system is created on one side of $\vec{r}$, and another one with $-\Phi_{0}$ is created on the side of $\vec{r}$. The adiabatic increase of the vector potential induces an elecric field, which generates Hall current. As a result, the charge $\pm \tilde{\sigma}_{x y}$ is induced where the flux tubes go through the system. We call the composite object of the charge and the flux tube as a "fluxon". Thus $f_{\alpha}(\vec{r})$ can be regarded as a creation operator of the fluxon-antifluxon pair. In fact, because $f_{\alpha}(\vec{r})$ creates an excited state with a fluxon-antifluxion pair, this process is actually non-adiabatic. However, we assume that, by increasing the vector potential sufficiently slowly, it does not create any excitation other than those required topologically, and is "adiabatic" in a generalized sense. Now, by further applying $f_{\alpha}\left(\vec{r}-l_{\bar{\alpha}} \vec{e}_{\bar{\alpha}}\right)$, we can move the fluxon by $-l_{\bar{\alpha}} \vec{e}_{\bar{\alpha}}$. Thus, the consecutive product

$$
\mathfrak{F}_{\alpha}(\vec{r}) \equiv \prod_{j=L_{\bar{\alpha}} / l_{\bar{\alpha}}}^{0} f_{\alpha}\left(\vec{r}-j l_{\bar{\alpha}}\right),
$$

when acted on the ground state $\left|\Psi_{0}\right\rangle$, creates a fluxonantifluxon pair, drags the fluxon along the fundamental cycle of the torus in $\bar{\alpha}$-direction, and pair-annihilate the fluxon and antifluxon. Thus the resulting state should be a ground state (vacuum), as long as the process is "adiabatic" in the extended sense discussed above. However, it should be also noted that $\mathfrak{F}_{\alpha}(\vec{r})$ leaves a string of modified vector potential behind the path, and thus the Hamiltonian after the process is not identical to the initial one. As in the case of $\mathcal{F}_{\alpha}$, this modified vector potential can be eliminated by a large gauge transformation, but it has to be squeezed onto the affected region. Namely, we define

$$
\mathfrak{U}_{\alpha}(\vec{r})=\exp \left(i \int d \vec{r}^{\prime} \theta_{\vec{r}}\left(\vec{r}^{\prime}\right) \rho\left(\vec{r}^{\prime}\right)\right),
$$

where

$$
\theta\left(\vec{r}^{\prime}\right)= \begin{cases}0 & r_{\alpha}^{\prime}<r_{\alpha} \\ 2 \pi \frac{r_{\alpha}^{\prime}-r_{\alpha}}{l_{\alpha}} & r_{\alpha} \leq r_{\alpha}^{\prime}<r_{\alpha}+l_{\alpha} \\ 2 \pi & r_{\alpha}+l_{\alpha}<r_{\alpha}^{\prime}\end{cases}
$$

Then

$$
\tilde{\mathfrak{F}}_{\alpha}(\vec{r}) \equiv \mathfrak{U}_{\alpha}(\vec{r}) \mathfrak{F}_{\alpha}(\vec{r})
$$

maps a ground state of the original Hamiltonian to a ground state of the same Hamiltonian.

Similarly to Eq. (34,

$$
T_{\alpha} \mathfrak{U}_{\alpha}(\vec{r}) T_{\alpha}^{-1} \mathfrak{U}_{\alpha}(\vec{r})^{-1}=e^{-\frac{2 \pi \mathrm{i}}{l_{\alpha}} \int_{r_{\alpha}<r_{\alpha}^{\prime} \leq r_{\alpha}+l_{\alpha}} d \vec{r}^{\prime} \rho\left(\vec{r}^{\prime}\right)}
$$

The integral in the exponent gives the total particle number within the strip $r_{\alpha}<r_{\alpha}^{\prime} \leq r_{\alpha}+l_{\alpha}$. It is not a conserved quantity like the total particle number in the entire system. However, assuming an incompressible liquid state, the particle number within the strip has only small fluctuations if $L_{\bar{\alpha}}$ is sufficiently larger than the correlation length of the pair correlation function (densitydensity correlation function). Thus it may be replaced as

$$
\int_{r_{\alpha}<r_{\alpha}^{\prime} \leq r_{\alpha}+l_{\alpha}} d \vec{r}^{\prime} \rho\left(\vec{r}^{\prime}\right) \sim \bar{\rho} l_{\alpha} L_{\bar{\alpha}} .
$$

Within this assumption,

$$
T_{\alpha} \mathfrak{U}_{\alpha}(\vec{r}) T_{\alpha}{ }^{-1} \mathfrak{U}_{\alpha}(\vec{r})^{-1} \sim e^{-2 \pi \mathrm{i} \bar{\rho} L_{\bar{\alpha}}} .
$$

Thus we expect

$$
T_{\alpha} \tilde{\mathfrak{F}}_{\alpha}(\vec{r}) T_{\alpha}{ }^{-1} \sim \tilde{\mathfrak{F}}_{\alpha}(\vec{r}) e^{-2 \pi \mathrm{i} \bar{\rho} L_{\bar{\alpha}}}
$$

when acting on the ground-state subspace.

Let us consider the system on a torus consisting of $L_{1} \times L_{2}$ unit cells, with the Landau gauge (5). As in Sec. IIIC, we take $L_{1}$ to be an integral multiple of $q$, and $L_{2}$ to be a coprime with $q$. Here we consider the 
"fluxon stitching" in 2-direction, $\mathfrak{F}_{1}\left(\vec{r}_{0}\right)$, with the starting point $\vec{r}_{0}$. In the present setup with the Landau gauge (5), the system is not invariant under $T_{1}$, and it is even impossible to define the magnetic translation operator in 1-direction. Nevertheless, as we have discussed in Sec. VIB, we can define an effective symmetry generator 44 on the ground-state subspace. Similar to Eq. 62,

$$
\mathcal{F}_{2}\left(\varphi \Phi_{0}\right) \mathfrak{U}_{1}(\vec{r})=\mathfrak{U}_{1}(\vec{r}) \mathcal{F}_{2}\left(\varphi \Phi_{0}\right) e^{2 \pi i \varphi \tilde{\sigma}_{x y}} .
$$

Combining Eq. (77) and Eq. (78) with $\varphi=\phi L_{2}$, we find

$$
\left(\tilde{\tilde{T}}_{1}\right)^{-1}\left[\tilde{\mathfrak{F}}_{1}(\vec{r})\right]^{-1} \tilde{\tilde{T}}_{1} \tilde{\mathfrak{F}}_{1}(\vec{r}) \sim e^{2 \pi i L_{2}\left(\phi \tilde{\sigma}_{x y}-\bar{\rho}\right)} \sim 1
$$

Now, both $\tilde{\mathfrak{F}}_{1}$ and $\tilde{\tilde{T}}_{1}$ maps the ground state to the ground state. Because of the uniqueness of the ground state (which was assumed), the phase factor appearing above should be unity. Thus we recover Theorem 1, using the fluxon stitching picture. We will see that this formulation also provides a useful insight for Fractional Quantum Hall effect.

\section{FRACTIONAL QUANTUM HALL EFFECTS}

\section{A. Fractional quantization of the Hall conductivity}

A FQHE must accompany a topological ground-state degeneracy on a torus [30]. As we have discussed in Section VB, we can choose "charge-density wave" ground state with respect to the direction under consideration, so that the total polarization of the ground in that direction is well-defined (expectation value of $\mathcal{U}_{1}$ does not vanish). In fact, in our setup with the Landau gauge (5), the system does not have the one-site translation symmetry $T_{1}$ in 1-direction. Thus we can expect that a chargedensity wave ground state in 1-direction is automatically chosen as the ground state of a generic finite-size system.

The commutation relation (65) then implies that, application of $\tilde{F}_{2}\left(\Phi_{0}\right)$ changes the expectation value of $\mathcal{U}_{1}$ by the phase factor $e^{2 \pi \mathrm{i} \tilde{\sigma}_{x y}}$. In other words, the total polarization $\overline{\mathcal{P}}_{1}$ is changed by $\tilde{\sigma}_{x y}$. This implies that, if $\tilde{\sigma}_{x y}$ is fractional and the system has a gap, then the new ground state obtained by the adiabatic process $\tilde{F}_{2}\left(\Phi_{0}\right)$ is different from the initial ground state. Thus, if the Hall conductivity takes a fractional value and the system has a gap above the ground states,

$$
\tilde{\sigma}_{x y}=\frac{\tilde{p}}{\tilde{q}}
$$

for coprimes $\tilde{p}$ and $\tilde{q}$, there must be $\tilde{q}$ degenerate, independent ground states. Namely, if there are (only) $n$ degenerate ground states below the gap, the Hall conductivity is quantized in unit of $(1 / n)\left(e^{2} / h\right)$. In other words, the ground state degeneracy $n$ must be an integral multiple of $\tilde{q}$.
This is the "non-edge" version of the argument by Tao and $\mathrm{Wu}[30]$. The present argument has the advantage particularly in the fact that the ground-state degeneracy is better defined in the absence of the gapless edge states.

\section{B. FQHE in a periodic system}

Now let us consider a many-particle system in a periodic potential or on a periodic lattice, with the total particle number conserved. As we have discussed in Section III B under the magnetic field, the particle number per the magnetic unit cell, $\bar{\rho} q$ is the relevant parameter for the LSM theorem. That is, if

$$
q \bar{\rho}=\frac{p^{\prime \prime}}{q^{\prime \prime}}
$$

is fractional with mutually coprime $p^{\prime \prime}$ and $q^{\prime \prime}$, the system must be either gapless or has $q^{\prime \prime}$-fold ground-state degeneracy.

Similarly to the case of the IQHE, we can obtain a stronger constraint based on the many-body polarization. As in Sec. VIB, we consider the system on the torus of the size $L_{1} \times L_{2}$ with the Landau gauge (5), and choose $L_{1}$ to be an integral multiple of $q q^{\prime}$ and $L_{2}$ to be a coprime with $q$ and $q^{\prime}$. Then Eq. 45 still holds. As in Sec. VII A, we assume that the system is an insulator in Resta-Sorella sense. That is, we can choose a complete set of the degenerate ground states, so that each of which has a non-vanishing expectation value $\left\langle\mathcal{U}_{1}\right\rangle$ with an asymptotically vanishing fluctuation of its argument, in the thermodynamic limit. Starting one particular ground state with a well-efined expectation value $\left\langle\mathcal{U}_{1}\right\rangle$, Eq. 45 implies that we can generate another ground state which has the total polarization $\mathcal{P}_{1}$ different from the original ground state by

$$
2 \pi L_{2}\left(\phi \tilde{\sigma}_{x y}-\bar{\rho}\right) .
$$

Since the total polarization is defined modulo $2 \pi$, and and we can choose $L_{2}$ as any integer, we can generate $\mathfrak{q}$ independent ground states (including the original one) if

$$
\phi \tilde{\sigma}_{x y}-\bar{\rho}=\frac{\mathfrak{p}}{\mathfrak{q}} .
$$

This implies that the number of the degenerate ground states, $n$, must be an integral multiple of $\mathfrak{q}$. Thus

$$
n\left(\phi \tilde{\sigma}_{x y}-\bar{\rho}\right) \in \mathbb{Z},
$$

or equivalently, using Eq. 81

$$
p \frac{\tilde{p}}{\tilde{q}}-\frac{p^{\prime \prime}}{q^{\prime \prime}} \in \frac{q}{n} \mathbb{Z} .
$$

As a corollary, this relation includes the fact that the ground-state degeneracy $n$ is an integral multiple of both $\tilde{q}$ and $q^{\prime \prime}$. The fact that $n$ is an integral multiple of $q^{\prime \prime}$ follows from the generalized LSM, and that $n$ is an integral multiple of $\tilde{q}$ follows from Ref. [30. However, the present result puts a stronger constraint in terms of the relation 84. 


\section{Fluxon stitching and braiding with background anyons}

Let us now discuss the fluxon stitching picture introduced in Sec. VIC, for the Fractional Quantum Hall states. All the discussions leading to Eq. (79) remain valid. The difference arises because the ground state is no longer unique, the phase factor appearing in Eq. 790 is not necessarily unity. In fact, it is generally not unity, and the nontrivial phase factor is related to the groundstate degeneracy, as we have just discussed in Sec. VIIB. On the other hand, the fluxon stitching picture provides an intuitive picture on the physical origin of the nontrivial phase factor.

That is, Eq. 79 can be rewritten as

$$
e^{2 \pi \mathrm{i} \bar{\rho} L_{2}} \sim\left(T_{1}\right)^{-1} \tilde{\mathfrak{F}}_{1} T_{1}\left(\tilde{\mathfrak{F}}_{1}\right)^{-1} \sim \mathcal{F}_{2} \tilde{\mathfrak{F}}_{1}\left(\mathcal{F}_{2}\right)^{-1}\left(\tilde{\mathfrak{F}}_{1}\right)^{-1}
$$

where we have used short-handed notation $\mathcal{F}_{2} \equiv$ $\mathcal{F}_{2}\left(\phi L_{2} \Phi_{0}\right)$ and $\tilde{\mathfrak{F}}_{1} \equiv \tilde{\mathfrak{F}}_{1}(\vec{r})$. The left-hand side of this equation can be interpreted as the probability amplitude of the process of moving the fluxon counter-clockwise along the closed loop as shown in Fig. 3. This closed loop encircles exactly one column of the torus in FIG. 3 . The adiabatic Berry phase acquired in this "fluxon braiding" process is $e^{2 \pi \mathrm{i} \bar{\rho} L_{2}}$ as directly computed in 777 , and it holds true irrespective of integer or fractional QHEs. On the other hand, the right hand side of (86) can be decomposed into two contributions:

$$
\begin{gathered}
\mathcal{F}_{2} \tilde{\mathfrak{F}}_{1}\left(\mathcal{F}_{2}\right)^{-1}\left(\tilde{\mathfrak{F}}_{1}\right)^{-1} \sim \\
{\left[\mathcal{F}_{2} \mathfrak{U}_{1}\left(\mathcal{F}_{2}\right)^{-1}\left(\mathfrak{U}_{1}\right)^{-1}\right] \cdot\left[\mathcal{F}_{2} \mathfrak{F}_{1}\left(\mathcal{F}_{2}\right)^{-1}\left(\mathfrak{F}_{1}\right)^{-1}\right]}
\end{gathered}
$$

The 1st contribution

$$
\mathcal{F}_{2} \mathfrak{U}_{1}\left(\mathcal{F}_{2}\right)^{-1}\left(\mathfrak{U}_{1}\right)^{-1} \sim e^{2 \pi \mathrm{i} \tilde{\sigma}_{x y} \phi L_{2}}
$$

can be understood as an Aharonov-Bohm phase, acquired when a fluxon carrying charge $\tilde{\sigma}_{x y}$ goes around $\phi L_{2}$ flux quanta piercing through each column of the torus in FIG.

3. This is true for both integer and fractional QHEs.

Meanwhile, the 2nd term $\mathcal{F}_{2} \mathfrak{F}_{1}\left(\mathcal{F}_{2}\right)^{-1}\left(\mathfrak{F}_{1}\right)^{-1}$ corresponds to nothing but the mutual braiding statistics between two particles 26, 43]: one is a fluxon $F$ and the other is the "background anyon" 27 contained in each column, as we will elaborate soon. In the case of Integer Quantum Hall states, no anyon exists and this braiding phase is unity, and therefore we recover 79 and Theorem 1. The nontrivial phase appearing in the case of a Fractional Quantum Hall state can be understood as follows.

Quite generally in a topologically ordered system on a lattice, in the presence of translational symmetry, the spatial arrangement of anyons in the ground state forms a translational-invariant periodic structure, known as fractionalization of translation symmetries (see Section VIII for details) [23, 24, 27, 44. In particular one (Abelian) anyon $a$ can be placed in each unit cell without breaking

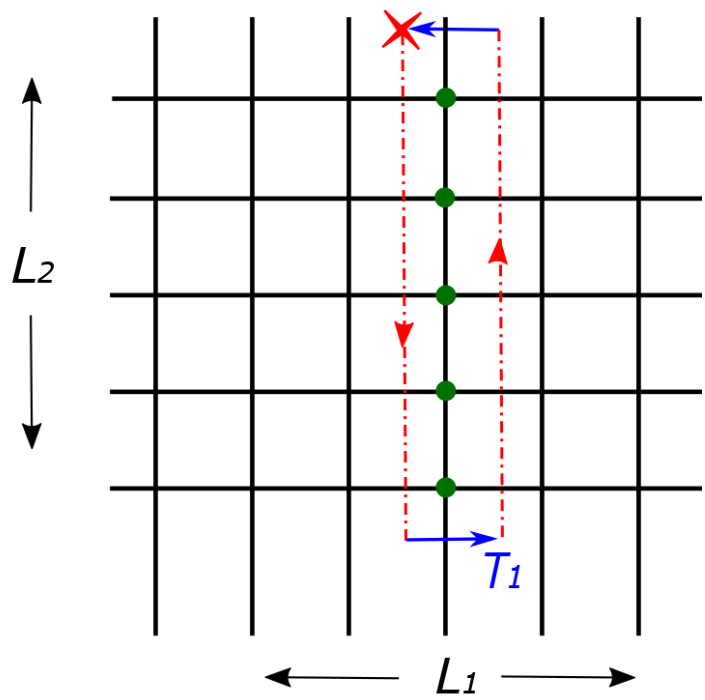

Figure 3: (color online) An illustration of the fluxon excitation (denoted by red cross) and the fluxon braiding process corresponding to Eq. (86).

lattice translational symmetry, which we refer to as the "background anyon" $a$. When another anyon $b$ travels around each unit cell counter-clockwise for once, it will acquire a braiding phase $e^{\mathrm{i} \theta_{b, a}}$ in this process. Since the fluxon $F$ goes around the array of the background anyons in a full column, this process picks up the phase factor

$$
\mathcal{F}_{2} \mathfrak{F}_{1}\left(\mathcal{F}_{2}\right)^{-1}\left(\mathfrak{F}_{1}\right)^{-1} \sim e^{\mathrm{i} \theta_{F, a} L_{2}}
$$

due to the fractional statistics between the fluxon and the background anyons. Here $\theta_{F, a} \in[0,2 \pi)$ represents the mutual statistical angle between fluxon $(F)$ and background anyon $(a)$ in each unit cell. Note that although distinct anyons can be permuted by translation symmetry 27, 45 47, their mutual statistics with fluxon $F$ should be invariant under translation so $\theta_{F, a}$ is welldefined.

Comparing Eqs. 866, (87) and 89, we find Theorem 2. Furthermore, comparing this with Eq. (84), we find the relation (27) between the statistical angle and the ground-state degeneracy.

In Appendix B we explicitly computed the Berry phase of the fluxon moving process in (86) in the effective Abelian Chern-Simons field theory, therefore establishing (86)- (89) in all Abelian topological orders. While such a field theory calculation does not apply to nonAbelian FQHEs, an alternative argument based on symmetry fractionalization algebra proves Theorem 2 for both Abelian and non-Abelian FQHEs, as we will show in the following section. 


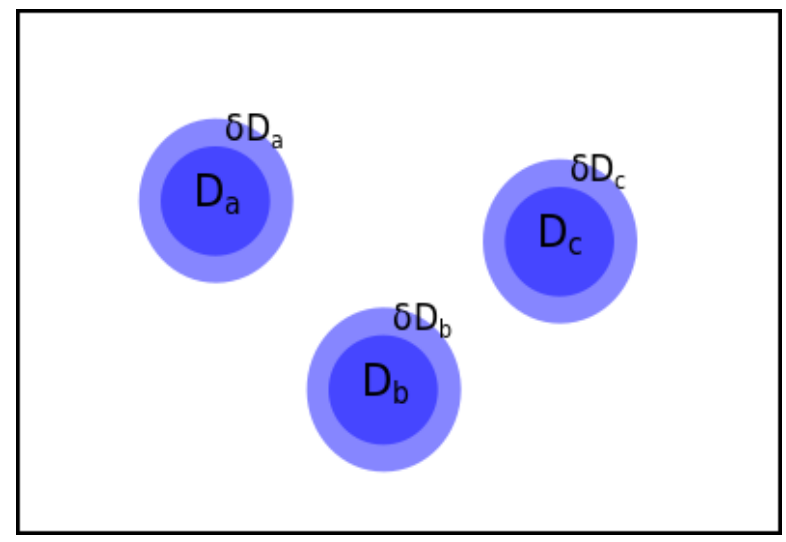

Figure 4: (color online) The fractionalization of global symmetries into spatially local regions.

\section{FRACTIONALIZATION ALGEBRA OF MAGNETIC TRANSLATION SYMMETRY}

In this Section, we discuss the problem from the viewpoint of symmetry fractionalizations [23, 24, 44], which gives a modern understanding of topological order. In particular, this is useful in describing systems with a nonAbelian topological order.

Let us consider a general gapped quantum phase (which may be topologically ordered) respecting a global symmetry group $S G$, and assume that the symmetry transformation would not exchange anyon types (i.e. superselection sectors) in the system. If one wants to understand how a global symmetry element $g \in S G$ transformes local excitations within finite spatial regions $D_{a}, D_{b}$ far apart from each other, one could decompose global symmetry transformation $R_{g}$ on the full manybody Hilbert space into a direct product of local symmetry actions $\Omega_{g}(a), \Omega_{g}(b) \ldots$ For example, $\Omega_{g}(a)$ only acts on a region $D_{a} \cup \delta D_{a}$, which is slightly larger than $D_{a}$ by a ribbon $\delta D_{a}$ (with a width $\sim$ correlation length) around the edge of $D_{a}$ (see Fig 4 ). These local operators $\Omega_{g}(a), \Omega_{g}(b) \ldots$ will be called as fractionalized symmetry operators below.

The basic assumption of symmetry fractionalization is the following condition [23, 24, 44]:

$$
R_{g}\left|\psi\left(D_{a}, D_{b} \ldots\right)\right\rangle=e^{i \theta_{g}} \cdot \Omega_{g}(a) \cdot \Omega_{g}(b) \ldots\left|\psi\left(D_{a}, D_{b} \ldots\right)\right\rangle,
$$

where $\left|\psi\left(D_{a}, D_{b} \ldots\right)\right\rangle$ describes any states whose quasiparticles are only localized within regions $D_{a} \cup D_{b} .$. , including the ground state. Here $e^{i \theta_{g}}$ is a possible $U(1)$ phase factor due to the background $g$-quantum-number outside the regions $D_{a} \cup D_{b} \ldots$ Importantly, $\theta_{g}$ is independent of the excitations inside regions $D_{a} \cup D_{b} \ldots$

Next, let us focus on one particular region, e.g. $D_{a}$. To save notation we denote this region as $D$ (and similarly denote the ribbon area around it as $\delta D$ ). Physically, for onsite unitary symmetry $g$, such as the $U(1)$ charge conservation, $\Omega_{g}$ on $D \cup \delta D$ can be obtained by the following adiabatic process. $g$ being onsite means that $R_{g}=\prod U_{g}(\vec{r})$ is a direct product of onsite symmetry operations. First, one creates a pair of $g$-symmetry defects in $\delta D$ and adiabatically braid them around $D$ along $\delta D$ then annihilate them (i.e., $\delta D$ can be viewed as the worldline of the $g$-defect). Second, one applies $\prod_{\vec{r} \in D} U_{g}(\vec{r})$ within the region $D$. The combination of these two operations well-defines the transformation of any excitated states in $D$, and sends the ground state back to the ground state (up to a $U(1)$ phase factor). One may use this combination as a construction of $\Omega_{g}$ for onsite symmetry $g$.

Note that by adiabatically creating $g$-symmetry defects, we mean adiabatically modifying the Hamiltonian such that there exist a string in the real space, like a branch cut. Any term in the Hamiltonian crossing the string is modified such that only the degrees of freedom on one side of the string is conjugated by $R_{g}$. The end points of the string are the $g$-symmetry defects. After braiding the $g$-symmetry-defect around $\delta D$, the final Hamiltonian is different from the original Hamiltonian by $\prod_{\vec{r} \in D} U_{g}^{-1}(\vec{r})$ conjugation, and applying $\prod_{\vec{r} \in D} U_{g}(\vec{r})$ would send this final Hamiltonian back to the original Hamiltonian.

For time-reveral and spatial symmetries, such as the (magnetic) translation symmetry studied here, similar defect-based construction of $\Omega_{g}$ becomes difficult. For instance, the symmetry-defect associated with translational symmetry is the dislocation, braiding which around a region involves adding/removing physical degrees of freedom. However, conceptually one still can accept the existence of $\Omega_{g}$ satisfying 90 quite generally (as long as $g$ is not changing the anyon types or superselection sectors). In fact, $\Omega_{g}$ for (magnetic) translational symmetries can be microscopically constructed if one employs certain formulations such as tensor-network or parton methods 48.

Below we assume that $\forall g$ in the magnetic translation symmetry group, $\Omega_{g}$ exists and they satisfy Eq. 90 . Note that in order to satisfy Eq. $\sqrt{90}, \Omega_{g}$ is not completely fixed. We already mentioned that $\Omega_{g}$ may include an overal $U(1)$ phase when acting on all excited states. But what is more important is that $\Omega_{g}$ may include a nontrivial phase factor dependent on the nature of the excitation: because the only modifiable action of $\Omega_{g}$ is within $\delta D$, which is spatially separated with the excitations, this dictates that the only nontrivial ambiguity is a braiding of an Abelian anyon $\epsilon(g)$ around $\delta D$ [24, 44].

Namely, combining $\Omega_{q}$ with an additional $\epsilon(g)$-anyon braiding: $\Omega_{g} \rightarrow \Omega_{g}^{\prime}=\epsilon(g) \cdot \Omega(g)$ can give another welldefined $\Omega_{g}^{\prime}$ action, which still satisfy Eq. 90 (after including the effect of $\epsilon(g)$ in other regions). Interestingly, when nontrivial symmetry fractionalization occurs, it is impossible to avoid the anyon braiding in the ambiguity of $\Omega_{g}$. Precisely, the symmetry fractionalization pattern in a gapped quantum phase is determined by the follow- 
ing algebra:

$$
\forall g_{1}, g_{2} \in S G, \Omega_{g_{1}} \cdot \Omega_{g_{2}}=\lambda\left(g_{1}, g_{2}\right) \Omega_{g_{1} \cdot g_{2}},
$$

where $\lambda\left(g_{1}, g_{2}\right) \in \mathcal{A}$ is an Abelian anyon and the Abelian group $\mathcal{A}$ is the fusion group of all Abelian anyons in the system. Associativity dictates that:

$$
\lambda\left(g_{1}, g_{2}\right) \cdot \lambda\left(g_{1} \cdot g_{2}, g_{3}\right)=\lambda\left(g_{2}, g_{3}\right) \cdot \lambda\left(g_{1}, g_{2} \cdot g_{3}\right) .
$$

Together with the $\epsilon\left(g_{1}\right), \epsilon\left(g_{2}\right), \epsilon\left(g_{3}\right)$ ambiguities, this indicates $\lambda\left(g_{1}, g_{2}\right) \in H^{2}(S G, \mathcal{A})$, i.e., $\lambda\left(g_{1}, g_{2}\right)$ is an element in the second cohomology group of $S G$ with coefficient in $\mathcal{A}$ (with trivial group action because we are considering the case with anyon types invariant under $S G)$.

Let us now apply this general framework to the magnetic translation group in the presence of global $U(1)$ charge conservation. The fundamental Eq.(9) indicates that:

$$
\Omega_{\tilde{T}_{1}} \Omega_{\tilde{T}_{2}} \Omega_{\tilde{T}_{1}}^{-1} \Omega_{\tilde{T}_{2}}^{-1}=\lambda_{a} \Omega_{U(\phi)},
$$

where $U(\phi) \equiv e^{2 \pi i \phi \hat{N}}$ is the global $U(1)$ rotation by angle $2 \pi \phi$. And $\lambda_{a}$ is the braiding of certain anyon $a$ around $\delta D$. This anyon- $a$ is appearing in the Theorem 2. Note that $\Omega_{\tilde{T}_{1}}$ and $\Omega_{\tilde{T}_{2}}$ have their overall $U(1)$ phases, $\epsilon\left(\tilde{T}_{1}\right)$ and $\epsilon\left(\tilde{T}_{2}\right)$ ambiguities, but the product on the LHS of Eq. (93) has all these ambiguities cancelled. This product and consequently the RHS, should be a completely fixed action on the ground state and excitations in the region D.

Although the RHS of Eq. 93 is completely determined, the anyon- $a$ and $\Omega_{U(\phi)}$ could have a relative ambiguity. Let us recall one definition of $\Omega_{U(\phi)}$, based on $U(\phi)$-defect construction. Because $U(1)$ group is continuous, this adiabatic construction can be made in a long length scale and low energy scale so that any irrelevant anyons will not be excited. Namely, the adiabatic insertion of a $\phi$-flux over a large area (but within $\delta D$ ), which is nothing but the $U(\phi)$-defect, should give a well-defined state. If we use this this natural definition of $\Omega_{U(\phi)}$, then $a$ is determined as long as $\phi$ is determined.

The subtlety is that in the global magnetic translation algebra Eq. (9), only the fractional part of $\phi$ is welldefined. Therefore, it is perfectly fine to use $\phi^{\prime}=\phi+1$ in the global magnetic translation. However, in the fractionalized operator $\Omega_{U(\phi)}$, adiabatically inserting a $\phi$-flux and $\phi^{\prime}$-flux over a large area would generally result in different defects. Clearly, $U\left(\phi^{\prime}\right)=U(\phi)+\lambda_{F}$ where $F$ is a particular anyon in the system which is created by adiabatically inserting $2 \pi$ flux over a large area. And $\lambda_{F}$ is the braiding operation of $F$ around $\delta D$. Consequently, because the RHS of Eq. 93 is fixed, if $\phi \rightarrow \phi+1$, then we must have $\lambda_{a} \rightarrow \lambda_{a-F}$ where $a-F$ is the anyon obtained by fusing $a$ and the antiparticle of $F$. This ambiguity has been discussed below Theorem- 2 .

Theorem 2 turns out to be just an application of Eq. (93). Let us consider an anyon $F$ sitting inside region
D. The LHS and RHS of Eq.93 describe two different actions on this state. Relative to the ground state, the RHS would pick up two phase factors: the braiding phase factor $\theta_{F, a}$ and the phase factor obtained by $\Omega_{U(\phi)}$. But $\Omega_{U(\phi)}$ is nothing but a measurement of the charge carried by $F$ by $\phi-U(1)$ rotation. Because $F$ must carry charge $\tilde{\sigma}_{x y}$, we know that this second phase factor is simply $e^{2 \pi \mathrm{i} \phi \tilde{\sigma}_{x y}}$.

Now let us look at the LHS, which magnetic translate the unit-fluxon- $F$ around one unit cell. The phase obtained in this process can be safely shown to be due to the charge density per unit cell: $e^{2 \pi \mathrm{i} \bar{\rho}}$. The details of the derivation can be found in Appendix C. Summarizing our results, we have obtained Theorem 2

$$
\begin{gathered}
e^{2 \pi \mathrm{i} \bar{\rho}}=\mathrm{LHS}=\mathrm{RHS}=e^{2 \pi \mathrm{i} \phi \tilde{\sigma}_{x y}+\mathrm{i} \theta_{F, a}} . \\
\text { IX. GENERALIZATION TO } \\
\text { NON-SYMMORPHIC LATTICES }
\end{gathered}
$$

Now let us generalize theorem 25 to a $2 \mathrm{~d}$ nonsymmorphic lattice with glide reflections. Among all 17 space groups in $2 \mathrm{~d}$ (wallpaper groups) only 4 are nonsymmorphic 49: $p g$ and $p m g$ with one glide plane, $p g g$ and $p 4 g$ with two perpendicular glide planes. In a $2 \mathrm{~d}$ system $\sigma_{x y}$ is odd under orientation-reversing glide operation, so we consider anti-unitary "magnetic glide" operation $\tilde{g}=\mathcal{U} \cdot g \cdot \boldsymbol{T}$ which includes a time reversal operation $\boldsymbol{T}$, where $\mathcal{U}$ is certain unitary transformation responsible for the magnetic translation algebra (9). Specifically we focus on the following magnetic algebra between magnetic glide $\tilde{g}_{1}$ and magnetic translation $\tilde{T}_{2}$

$$
\tilde{T}_{2} \tilde{g}_{1} \tilde{T}_{2}\left(\tilde{g}_{1}\right)^{-1}=e^{-\mathrm{i} \pi \phi \hat{N}}
$$

where $\hat{N}=\sum_{\mathbf{r}} \hat{N}_{\mathbf{r}}$ is the total charge of the system. Choosing a Landau gauge where $\tilde{T}_{2}=T_{2}$ is the usual translation along $\vec{a}_{2}$ direction, we have

$$
\begin{gathered}
\tilde{g}_{1} \equiv e^{\mathrm{i} \pi \phi \sum_{\mathbf{r}} r_{2} \hat{N}_{\mathbf{r}}} \cdot g_{1} \cdot \boldsymbol{T} \\
\left(r_{1}, r_{2}\right) \stackrel{g_{1}}{\longrightarrow}\left(r_{1}+1 / 2,-r_{2}\right), \quad\left(\tilde{g}_{1}\right)^{2}=\tilde{T}_{1} .
\end{gathered}
$$

where $\mathbf{r}=r_{1} \vec{a}_{1}+r_{2} \vec{a}_{2}$ again denotes the center of mass position for each unit cell. It's a convenient representation of magnetic translation (9) on a $2 \mathrm{~d}$ non-symmorphic lattice, with $\phi$ flux quanta in each unit cell.

Completely in parallel to theorems (25) and (26), we can obtain the following relation based on fractionalization algebra of 95 acting on a single fluxon $F$ :

$$
e^{\mathrm{i} \frac{\bar{\rho}}{2}}=e^{\mathrm{i} \tilde{\sigma}_{x y} \frac{\phi}{2}} \cdot e^{\mathrm{i} \theta_{F, a}}
$$

The difference between the above relation and (94) of the magnetic translation case can be intuitively understood as the following. Magnetic glide operation $\tilde{g}_{1}$ can be viewed as a half of a magnetic translation $\tilde{T}_{1}$, dividing each unit cell into two halves. With glide symmetry, the 
charge density $\bar{\rho} / 2$ and flux density $\phi \Phi_{0} / 2$ in each half of a unit cell becomes well defined, and so is the background anyon $a$ in each half of the unit cell. According to (97), we immediately prove the following theorem

Theorem 3. Filling-enforced constraint for QHE on non-symmorphic lattices: Hall conductivity of a gapped 2d insulator must satisfy

$$
\bar{\rho}=\sigma_{x y} \cdot \phi+\frac{\theta_{F, a}}{\pi} \bmod 2 .
$$

if it preserves magnetic algebra 950 on a nonsymmorphic lattice preserving glide symmetry 96).

An immediate corollary for $\phi=0$ is that for a generic system preserving magnetic glide symmetry (96), i.e. the combination of glide $g_{1}$ and time reversal $\boldsymbol{T}$ (where arguments in Ref. 50 does not apply), charge density $\bar{\rho}$ per u.c. must be an even integer to achieve a non-fractionalized featureless insulator.

\section{APPLICATIONS}

\section{A. IQHE models}

Theorems 1 and 2 directly apply to integer and fractional QHEs on lattices, i.e. integer and fractional Chern insulators. We first discuss applications of Theorem 1 to IQHEs with a unique ground state on a torus.

The Harper-Hofstadter models [4, 51] which study the motion of Bloch electrons in a magnetic field is a simple realization of nontrivial IQHE [5]. While Theorem 1 was proved earlier in Ref. [20, apparently its consequences has not been much appreciated. $\pi$-flux models $\left(\phi=\frac{1}{2}\right)$ of spinless particles at half filling (half charge per lattice site) has been studied in various cases. Theorem 1 dictates that with $\bar{\rho}=\frac{1}{2}$ per unit cell, e.g. on square, triangular and kagome lattices, any unique ground state must have an odd Hall conductance $\tilde{\sigma}_{x y}=1 \bmod 2$. In particular, $\tilde{\sigma}_{x y}$ cannot vanish in this case, even though the time-reversal symmetry of $\pi$-flux models seems to allow $\tilde{\sigma}_{x y}=0$. Of course it is still possible to have $\tilde{\sigma}_{x y}=0$ in the $\pi$-flux models. However, for that there must be degenerate ground states. In other words, a trivial insulator at half filling is forbidden. Now with our proof in the present paper, this statement also applies to interacting systems.

In honeycomb-lattice $\pi$-flux models at half filling, we have $\bar{\rho}=1$. Then Theorem 1 requires an even Hall conductance, provided that the ground state is unique. This is indeed the case for IQH states of free fermions [52. In fact, our proof is valid also for interacting bosons, and requires an even Hall conductance for a bosonic model on the honeycomb lattice with $\pi$-flux. This is indeed consistent with the finding of a recent work [53].

\section{B. Magnetically ordered systems}

Another less obvious application is magnetically ordered systems, which are invariant under the combination of lattice translations and spin rotations. Prominent examples include the chiral spin density wave states [54, 55. in $\frac{1}{2}$-doped triangular lattice systems and $\frac{1}{4}$-doped honeycomb lattice systems, where $\bar{\rho}=1 / 2(\bmod 1)$ per unit cell. Their magnetic orders preserve magnetic translation

$$
\tilde{T}_{1,2}=\left(\mathrm{i} \hat{n}_{1,2} \cdot \vec{\sigma}\right) T_{1,2}, \hat{n}_{1} \cdot \hat{n}_{2}=0 \Longrightarrow\left\{\tilde{T}_{1}, \tilde{T}_{2}\right\}=0
$$

Microscopically the spin chirality effectively provides a $\pi$ flux $(\phi=1 / 2)$ per unit cell, and the electronic ground state supports Chern bands. But due to theorem (25), we know that $\sigma_{x y}$ must be an odd integer independent of microscopic details.

\section{Fractional Quantum Hall States on lattice}

Next we discuss the application of (26) to lattice FQHEs. Once we specify the topological order in the bulk, anyon statistics $\theta_{F, a}$ can only take certain discrete values and (26) can provide a strong constraint on the Hall conductivity. As a demonstration we work out two examples explicitly: $\nu=1 / 2$ Laughlin state and $Z_{2}$ topological order (toric code). According to Theorem 2 they all satisfy the following conditions:

$$
\bar{\rho}=\tilde{\sigma}_{x y} \phi+\frac{\theta_{F, a}}{2 \pi} \quad \bmod 1, \quad \tilde{\sigma}_{x y}=\frac{\theta_{F, F}}{2 \pi} \quad \bmod 1 .
$$

First let's consider $\nu=1 / 2$ Laughlin state which hosts quasiparticle $s$ obeying semion statistics. The only nontrivial mutual statistics is $\theta_{s, s}=\pi$ and hence there are two different situations. First scenario corresponds to familiar chiral spin liquids [56] and fractional Chern insulators, where the fluxon is a semion $F \sim s$ and hence Hall conductance is a half integer

$$
\tilde{\sigma}_{x y}=\frac{1}{2} \bmod 1 .
$$

Without loss of generality, we consider $\tilde{\sigma}_{x y}=1 / 2$ and in this case we have

$$
\bar{\rho}=\frac{1}{2} \phi+\frac{\theta_{s, a}}{2 \pi} \quad \bmod 1 \Longrightarrow \bar{\rho}=\frac{1}{2} \phi \quad \bmod \frac{1}{2}
$$

In a usual lattice model with translational symmetry $(\phi=0), \bar{\rho}=\frac{\theta_{s, a}}{2 \pi} \bmod 1$ and therefore only half filling $\bar{\rho}=\frac{1}{2} \bmod 1$ and integer filling are compatible to a $\nu=\frac{1}{2}$ Laughlin state.

In the 2nd scenario the fluxon is a boson $(F \sim 1)$, leading to an integer Hall conductance. Since $\theta_{1, a}=0$, we have relation

$$
\bar{\rho}=\tilde{\sigma}_{x y} \phi \quad \bmod 1, \quad \tilde{\sigma}_{x y} \in \mathbb{Z} .
$$


It can simply be realized in a system where chargeneutral (bosonic) particle-hole excitations form a $\nu=1 / 2$ Laughlin state on top of charged background. In this scenario only an integer filling is compatible.

Next we comment on toric-code-type 57 $Z_{2}$ topological order. As we will show below, our result has an interesting consequence even when there is no magnetic field and the Hall conductivity vanishes. The $Z_{2}$ topological phase has 3 types of anyons $\{e, m, \epsilon\}$, which obey mutual semion statistics $\theta_{e, m}=\theta_{e, \epsilon}=\theta_{m, \epsilon}=\pi$ between distinct anyons. In this theory, all anyons have a trivial self braiding angle $\theta_{a, a}=0$. This implies $\theta_{F, F}=0$ and thus $\tilde{\sigma}_{x y} \in \mathbb{Z}$ because of Eq. 29.

$$
\bar{\rho}=\tilde{\sigma}_{x y} \phi+\frac{\theta_{F, a}}{2 \pi} \bmod 1
$$

In a usual lattice model with $\phi=0$, this further leads to $\bar{\rho}=\frac{\theta_{F, a}}{2 \pi} \bmod 1$. This means that at half filling, both the background anyon and the fluxon are nontrivial anyons, and they cannot be of the same type. This is exactly the situation for $Z_{2}$ spin liquids in a half-filled Mott insulator, where $F \sim m$ and $a \sim e$.

\section{CONCLUSIONS AND DISCUSSIONS}

In this work, we have studied quantum Hall effect in two-dimensional periodic potentials or periodic lattices. We formulate and prove a universal relation among the quantized Hall conductivity, the charge and flux densities per the physical unit cell, and the possible anyon statistics: Eq. 25 for IQHE, Eq. 26) for FQHE, and Eq. 987 for a non-symmmorphic lattice with a glide symmetry. This serves as a strong constraint on the possible gapped insulating ground state of the system. While our work may be regarded as a combination of the Laughlin's gauge argument and the LSM theorem, our result gives a stronger constraint than what can be obtained by applying each of these known results independently. In particular, according to the standard generalization of the LSM theorem, a featureless insulator without fractionalization would be possible even for a fractional filling (i.e. fractional number of particles per physical unit cell) if the number of particle per magnetic unit cell is integer. Nevertheless, our result implies that these insulators cannot be a trivial Mott insulator and must have a nonvanishing Hall conductivity.

Thus, our results constitute useful guides for the search of desired integer and fractional QH states in a generic many-body system with magnetic translational symmetries. They provide strong constraints on the possible gapped ground states at a given filling number and a given magnetic flux density. They can be applied to Harper-Hofstadter models and certain magnetically ordered systems.

Our result follows from large gauge invariance and magnetic translation symmetry, is applicable to general interacting quantum many-particle systems in periodic potentials. We developed several different approaches, which are complementary to each other: (i) a cut and glue proof relying on edge states, presented in Section IV] (ii) a pure bulk proof based on the polarization operator, presented in Section VIA (iii) arguments based on the Berry phase of moving a $\Phi_{0}$ fluxon around a closed loop, presented in Sections VIC and VIIC, (iv) arguments based on the symmetry fractionalization algebra of a $\Phi_{0}$ fluxon, presented in Section VIII. Each of these arguments may be also useful in exploring other issues in quantum many-body problem.

In this work we have focused on insulators with a global $U(1)$ charge conservation in two spatial dimensions. Magnetic translations can also be defined for a discrete global symmetry. In an outlook for the future, it will be interesting to generalize the framework to systems with other global symmetries, in other words symmetry protected and enriched topological phases [58]. Another direction is to generalize this work to other spatial dimensions.

As a very interesting development after the present paper appearing in arXiv, Matsugatani et al. [59] proposed an alternative formulation of the present result in terms of many-body Chern number, and derived stronger constraints in the presence of crystal symmetries.

\section{Acknowledgments}

We thank Gil-Young Cho, Eduardo Fradkin, Miklos Lajko, Sid Parameswaran, Nick Read, T. Senthil, Kirill Shtengel, and Haruki Watanabe for useful discussions. We thank Aspen Center for Physics and Kavli Institute for Theoretical Physics, UC Santa Barbara, for hospitality, where parts of this work were performed under support of NSF grants PHY-1066293 and PHY-1607611, and NSF grants PHY-1125915 and PHY1748958, respectively. This work is also supported by NSF under award No. DMR-1653769 (YML) and DMR1151440 (YR), and by MEXT/JSPS KAKENHI Grants Nos. JP16K05469, JP17H06462, and JP19H01808 (MO).

\section{Appendix A: Charge pumping and generalized Luttinger's theorem for edge states of Abelian QHE}

First we prove the relation 49 between charge densities on the edge of two neighboring cylinders. Assuming the bulk has a unique ground state without topological order, its low-energy long-wavelength physics can be captured by a multi-component Abelian Chern-Simons theory 31

$$
\mathcal{L}_{\text {bulk }}=\frac{\epsilon^{\mu \nu \rho}}{4 \pi} \sum_{I, J} \mathbf{K}_{I, J} a_{\mu}^{I} \partial_{\nu} a_{\rho}^{J}-\frac{\epsilon^{\mu \nu \rho}}{2 \pi} A_{\mu} \sum_{I} \mathbf{q}_{I} \partial_{\nu} a_{\rho}^{I}(\mathrm{~A} 1)
$$

where $a_{\mu}^{I}$ are dynamical gauge fields describing bulk excitations. $\mathbf{K}$ is an integer-valued non-singular symmetric 
matrix satisfying $|\operatorname{det} \mathbf{K}|=1$, and $\mathbf{q}$ is an integer-valued vector called "charge vector". Gauge invariance on an open manifold leads to the following effective edge theory for the left edge along $\vec{a}_{2} \| \hat{y}$-direction

$$
\begin{gathered}
\mathcal{L}_{\text {L-edge }}=\sum_{I, J} \frac{\mathbf{K}_{I, J}}{2 \pi} \partial_{t} \phi_{I} \partial_{y} \phi_{J} \\
+\sum_{I} \mathbf{q}_{I} \frac{A_{0} \partial_{y} \phi_{I}-A_{y} \partial_{0} \phi_{I}}{2 \pi}
\end{gathered}
$$

where $\phi_{I}(y, t)$ are chiral boson fields which describes lowenergy dynamics of the left edge, satisfying the current algebra

$$
\left[\phi_{I}\left(y^{\prime}\right), \partial_{y} \phi_{J}(y)\right]=2 \pi \mathrm{i} \mathbf{K}_{I, J}^{-1} \delta\left(y-y^{\prime}\right)
$$

The charge density on the edge is given by

$$
\rho(y)=\frac{1}{2 \pi} \sum_{I} \mathbf{q}_{I} \partial_{y} \phi_{I}(y)
$$

As shown in FIG. 1 the two neighboring cylinders are related by $\left(\tilde{T}_{1}\right)^{L_{1}}$ operation, which includes a large gauge transformation

$$
\begin{aligned}
& \mathcal{U}_{2}^{\phi l_{1} L_{2}} \equiv e^{\mathrm{i} 2 \pi \phi L_{1} \sum_{\mathbf{r}} r_{2} \hat{N}_{\mathbf{r}}} \\
& =\exp \left[\mathrm{i} 2 \pi \phi L_{1} \int y \rho(y) \mathrm{d} y\right]
\end{aligned}
$$

in addition to pure lattice translations. As a result we have

$$
\begin{gathered}
\rho_{n+1, L}(y)=\mathcal{U}_{2, n}^{\phi l_{1} L_{2}} \rho_{n, L}(y) \mathcal{U}_{2, n}^{-\phi l_{1} L_{2}} \\
=\rho_{n, L}(y)-\phi l_{1} \cdot \sigma_{x y}, \quad \sigma_{x y}=\mathbf{q}^{T} \mathbf{K}^{-1} \mathbf{q} .
\end{gathered}
$$

where $\mathcal{U}_{2, n}$ denotes an elementary large gauge transformation acting on left edge of $n$-th cylinder. We've used the current algebra A3 in the derivation. Similar derivations can be made for the right edge, where the change of charge density is opposite to the left edge.

This is the well-known chiral anomaly 32: i.e. chiral edge modes of QHEs is not invariant under large gauge transformations. The chiral anomaly of edge states is canceled 31] by the anomaly of bulk Chern-Simons theory A1, so that the entire system is still gauge invariant.

Next we prove the generalized Luttinger's theorem (48) for the edge states of a $2 \mathrm{~d}$ Abelian insulator. It's straightforward to verify from current algebra $\mathrm{A} 3$ that the total momentum operator on the edge is written as

$$
P_{y}=\sum_{I, J} \frac{\mathbf{K}_{I, J}}{4 \pi} \int \mathrm{d} y \partial_{y} \phi_{I} \partial_{y} \phi_{J}
$$

satisfying

$$
\left[\phi_{I}(y), P_{y}\right]=\mathrm{i} \partial_{y} \phi_{I}(y)
$$

Now let's insert a flux quantum $\Phi_{0}$ through the cylinder. It's implemented by large gauge transformation $\mathcal{U}_{1 / L_{2}}$ and it's straightforward to see that

$$
\mathcal{U}_{2} P_{y} \mathcal{U}_{2}^{-1}=P_{y}+2 \pi \int_{0}^{L_{2}} \frac{\mathrm{d} y}{L_{2}} \rho(y)
$$

Therefore we've proved the generalized Luttinger's theorem (48) for the edge states of a gapped bulk.

\section{Appendix B: Berry phase of fluxon braiding in Abelian QHE}

In this section we compute the Berry phase obtained by adiabatically dragging a $\Phi_{0}$ fluxon $F$ around a closed contour $\mathcal{C}$ enclosing certain anyon excitations. For any Abelian topological order, the effective Lagrangian describing this adiabatic process is 31

$$
\mathcal{L}_{0}=\frac{\epsilon^{\mu \nu \rho}}{4 \pi} \sum_{I, J} \mathbf{K}_{I, J} a_{\mu}^{I} \partial_{\nu} a_{\rho}^{J}-\sum_{I} a_{\mu}^{I}\left(\frac{\epsilon^{\mu \nu \rho}}{2 \pi} \mathbf{q}_{I} \partial_{\nu} A_{\rho}+j_{I}^{\mu}\right)
$$

where

$$
A_{\mu}(\mathbf{r}, t)=\delta A_{\mu}(\mathbf{r}, t)+\bar{A}_{\mu}(\mathbf{r})
$$

is the external (classical) EM vector potential. The timeindependent piece $\bar{A}_{\mu}(\mathbf{r})$ describes a uniform background magnetic field of strength $\phi \Phi_{0}$ per unit cell (we've chosen the area of unit cell to be unity for simplicity) as shown in (B6), while the time-dependent piece $\delta A_{\mu}(\mathbf{r}, t)$ is introduced to adiabatically move a fluxon as shown in B5.

Meanwhile $a_{\mu}^{I}$ are dynamical gauge fields describing bulk excitations. $\mathbf{K}$ is an integer-valued non-singular symmetric matrix and $\mathbf{q}$ is an integer-valued vector called "charge vector". The conserved $U(1)$ charge current in $2+1$-D is given by

$$
J_{e}^{\mu}=\frac{\epsilon^{\mu \nu \rho}}{2 \pi} \sum_{I} \mathbf{q}_{I} \partial_{\nu} a_{\rho}^{I}
$$

Meanwhile

$$
j_{I}^{\mu}(\mathbf{r})=\delta_{\mu, 0} j_{I}^{0}(\mathbf{r})=\delta_{\mu, 0} \sum_{\alpha_{I}=1}^{N_{I}} \delta\left(\mathbf{r}-\mathbf{r}_{\alpha_{I}}\right) .
$$

are time-independent classical fields describing the background anyons located at $\left\{\mathbf{r}_{\alpha_{I}} \mid 1 \leq \alpha_{I} \leq N_{I}\right\}$ in the ground state. The fractional statistics of bulk anyon excitations are encoded by the $\mathbf{K}$ matrix in (B1). In the adiabatic process, flux insertion and movement won't change the original background anyon $j_{I}^{\mu}(\mathbf{r})$. Adiabatic dragging a fluxon along a closed contour $\mathcal{C}$ is implemented by a time-dependent classical vector potential $A_{\mu}(\mathbf{r}, t)$ satisfying

$$
\begin{gathered}
\epsilon^{0 \mu \nu} \partial_{\mu} \delta A_{\nu}(\mathbf{r}, t)=2 \pi \delta^{2}\left(\mathbf{r}-\mathbf{r}_{0}(t)\right) \\
\epsilon^{0 \mu \nu} \partial_{\mu} \bar{A}_{\nu}(\mathbf{r})=\phi
\end{gathered}
$$

where the fluxon located at $\mathbf{r}_{0}(t) \in \mathcal{C}$ goes along closed contour $\mathcal{C}$ once in time period $0 \leq t \leq T$. The Berry phase acquired in this process can be obtained as

$$
\int_{0}^{T} \mathrm{~d} t \int \mathrm{d}^{2} \mathbf{r} A_{\mu}(\mathbf{r}, t) J_{e}^{\mu}=2 \pi \int_{\mathbf{r} \in \mathcal{C}} \mathrm{d}^{2} \mathbf{r} J_{e}^{0}=2 \pi \rho_{\in \mathcal{C}}
$$

which is nothing but $2 \pi$ times the total charge number in the area enclosed by $\mathcal{C}$. This corresponds to the l.h.s. 
of Eq. $86, e^{2 \pi \mathrm{i} \bar{\rho} L_{2}}$ when the contour encloses $L_{2}$ unit cells in one column of the torus.

We can also obtain this Berry phase from the effective response theory for external EM vector potential $\delta A_{\mu}$, by integrating out all bulk excitations captured by Abelian gauge fields $\left\{a_{\mu}^{I}\right\}$. This will lead to a Chern-Simons term of the following form

$$
\begin{gathered}
\prod_{I} \int \mathcal{D} a_{\mu}^{I} e^{-\int \mathrm{d} t \mathrm{~d}^{2} \mathbf{r} \mathcal{L}_{0}}=e^{-\int \mathrm{d} t \mathrm{~d}^{2} \mathbf{r} \mathcal{L}_{\mathrm{eff}}} \Longrightarrow \\
\mathcal{L}_{e f f}=-\frac{\epsilon^{\mu \nu \rho}}{4 \pi} \sum_{I, J} \mathbf{K}_{I, J}^{-1} \cdot \\
\left(\mathbf{q}_{I} A_{\mu}-2 \pi \frac{\epsilon_{\mu \alpha \beta}}{\partial^{2}} \partial^{\alpha} j_{I}^{\beta}\right) \partial_{\nu}\left(\mathbf{q}_{J} A_{\rho}-2 \pi \frac{\epsilon_{\rho \gamma \delta}}{\partial^{2}} \partial^{\gamma} j_{J}^{\delta}\right) \\
=-\frac{\sigma_{x y}}{4 \pi} \epsilon^{\mu \nu \rho} \delta A_{\mu} \partial_{\nu} \bar{A}_{\rho}-\sum_{I} \mathbf{q}_{I} \mathbf{K}_{I, J}^{-1} \delta A_{\mu} j_{J}^{\mu}, \\
\sigma_{x y}=\mathbf{q}^{T} \mathbf{K}^{-1} \mathbf{q} .
\end{gathered}
$$

where we've used relation

$$
\partial_{\mu} j^{\mu}=0 \Longrightarrow \epsilon^{\mu \nu \rho} \partial_{\nu} d_{\rho}=j^{\mu}, \quad d_{\mu}=-\epsilon_{\mu \nu \rho} \frac{\partial^{\nu}}{\partial^{2}} j^{\rho} .
$$

Note that in 88 the Hopf term 60

$$
\begin{gathered}
\mathcal{L}_{\text {Hopf }}=-\pi \sum_{I, J} \epsilon^{\mu \nu \rho} d_{\mu}^{I} \mathbf{K}_{I, J}^{-1} j_{J}^{\mu} \\
=-\pi \sum_{I, J} \epsilon^{\mu \nu \rho} d_{\mu}^{I} \mathbf{K}_{I, J}^{-1} \partial_{\nu} d_{\rho}^{J} \\
=-\pi \sum_{I, J} \epsilon_{\mu \nu \rho} j_{I}^{\mu} \mathbf{K}_{I, J}^{-1} \frac{\partial^{\nu}}{\partial^{2}} j_{J}^{\rho}
\end{gathered}
$$

vanishes for stationary background anyons $j_{I}^{\mu}(\mathbf{r})=$ $\delta_{\mu, 0} j_{I}^{0}(\mathbf{r})$. The 1 st term in $\mathrm{B} 8$

$$
\mathcal{L}_{A B}=-\frac{\sigma_{x y}}{4 \pi} \epsilon^{\mu \nu \rho} \bar{A}_{\mu} \partial_{\nu} \delta A_{\rho}
$$

can be understood as Aharonov-Bohm phase 88 between polarization charge $\frac{\epsilon^{\mu \nu \rho}}{2 \pi} \partial_{\nu} A_{\rho}$ near flux core $\mathbf{r}_{0}(t)$ and background flux 61. Compared to Hopf term (B10), the 2nd term in $\mathrm{B} 8$

$$
\begin{gathered}
\mathcal{L}_{F, a}=-\sum_{I, J} \mathbf{q}_{I} \mathbf{K}_{I, J}^{-1} \delta A_{\mu} j_{J}^{\mu} \\
=-2 \pi \sum_{I, J} \epsilon^{\mu \nu \rho} d_{\mu}^{I} \mathbf{K}_{I, J}^{-1} \frac{\partial_{\nu} \delta A_{\rho}}{2 \pi} \mathbf{q}_{J}
\end{gathered}
$$

is nothing but mutual braiding statistics $(89)$ between fluxon (labeled by quasiparticle current $\mathbf{q}_{I} \frac{\epsilon^{\mu \tau p}}{2 \pi} \partial_{\nu} A_{\rho}$ ) and background anyons (quasiparticle current $j_{I}^{\mu}$ ).

If the bulk has a unique gapped ground state (no topological order), we have $|\operatorname{det} \mathbf{K}|=1$ and hence the 2 nd term is always a multiples of $2 \pi$. Thus for Abelian topological orders, we've established the equality between total phase factor on the l.h.s. of (86), and the sum of $\mathrm{AB}$ phase 88 and anyon braiding phase 89 on the r.h.s. of $(86)$.

Notice that in the effective field theory $(\mathrm{B} 8)$ describing the long-wavelength response of the system to fluxons:

$$
\mathcal{L}_{e f f}=-\delta A_{\mu} \sum_{I} \mathbf{q}_{I} \mathbf{K}_{I, J}^{-1}\left[\frac{\epsilon^{\mu \nu \rho}}{4 \pi} \mathbf{q}_{J} \bar{A}_{\rho}+j_{J}^{\mu}\right]
$$

there is an apparent ambiguity on the definition of (timeindependent) background vector potential $\bar{A}_{\mu}$ and background anyon density $j_{J}^{\mu}$, since we can always redefine them in the following way

$$
\bar{A}_{\mu} \rightarrow \bar{A}_{\mu}+\alpha_{\mu}, \quad j_{J}^{\mu} \rightarrow j_{J}^{\mu}-\mathbf{q}_{J} \alpha_{\mu} .
$$

without changing the effective Lagrangian $(\bar{B} 13)$ at all. This is exactly the ambiguity discussed in (28), where the background flux density increase by one flux quantum per unit cell, while the background anyon density changes by removing one fluxon $-F$ per unit cell.

\section{Appendix C: Magnetically translating a unit fluxon}

Our goal in this section is to establish:

$$
\Omega_{\tilde{T}_{1}}(F) \Omega_{\tilde{T}_{2}}(F) \Omega_{\tilde{T}_{1}}^{-1}(F) \Omega_{\tilde{T}_{2}}^{-1}(F)=e^{2 \pi i \bar{\rho}},
$$

where $\Omega_{\tilde{T}_{i}}(i=1,2)$ are the fractionalized magnetic translation operators acting on the region $D$, and $\Omega_{\tilde{T}_{i}}(F)$ describes the action of this operator on the unit-fluxon- $F$ located inside $D$. Here by definition, the unit-fluxon- $F$ is a long-length-scale, low-energy object which is created by adiabatically inserting $2 \pi$ magnetic flux over a large area inside $D$.

Let us denote the unknown phase factor $\Omega_{\tilde{T}_{1}}(F) \Omega_{\tilde{T}_{2}}(F) \Omega_{\tilde{T}_{1}}^{-1}(F) \Omega_{\tilde{T}_{2}}^{-1}(F)$ as $\eta$. To reach our goal, we firstly show that the phase $\eta$ from this discrete algebra can be computed by an adiabatic Berry's phase corresponding to transporting the unit-fluxon- $F$ around a unit cell. Second, this adiabatic Berry's phase will be computed to be $e^{2 \pi i \bar{\rho}}$.

By locality and the defining property Eq. 90 , the conjugation transformation of any local quantum operator $\hat{O}(D)$ acting within region $D$ by fractionalized operators $\Omega_{\tilde{T}_{i}}$ is the same as the conjugation transformation by the global operator $\tilde{T}_{i}$. Namely:

$$
\Omega_{\tilde{T}_{i}} \hat{O}(D) \Omega_{\tilde{T}_{i}}^{-1}=\tilde{T}_{i} \hat{O}(D) \tilde{T}_{i}^{-1}, i=1,2 .
$$

From now on we will adopt the following notation for conjugation transformation:

$$
\tilde{T}_{i} \hat{O}(D) \equiv \tilde{T}_{i} \hat{O}(D) \tilde{T}_{i}^{-1} .
$$

Let us denote a quantum state with one fluxon- $F$ inside $\mathrm{D}$ as $|F\rangle$. To be precise, $|F\rangle$ also hosts an antifluxon somewhere far awary from $D$. We define $\Omega_{\tilde{T}_{i}}|F\rangle \equiv$ $\left|\Omega_{\tilde{T}_{i}} F\right\rangle$. Consider any two local quantum operators $\hat{O}_{1}$, and $\hat{O}_{2}$ such that the following quantum amplitudes are nonvanishing:

$$
\left\langle\Omega_{\tilde{T}_{1}} F\left|\hat{O}_{1}\right| F\right\rangle \neq 0 ; \quad\left\langle\Omega_{\tilde{T}_{2}} F\left|\hat{O}_{2}\right| F\right\rangle \neq 0 .
$$

From Eq. (C2), we then know that the relations between quantum amplitudes:

$$
\begin{aligned}
& \left\langle\Omega_{\tilde{T}_{1}} F\left|\hat{O}_{1}\right| F\right\rangle=\left\langle\left.\Omega_{\tilde{T}_{2}} \Omega_{\tilde{T}_{1}} F\right|^{\tilde{T}_{2}} \hat{O}_{1} \mid \Omega_{\tilde{T}_{2}} F\right\rangle, \\
& \left\langle\Omega_{\tilde{T}_{2}} F\left|\hat{O}_{2}\right| F\right\rangle=\left\langle\left.\Omega_{\tilde{T}_{1}} \Omega_{\tilde{T}_{2}} F\right|^{\tilde{T}_{1}} \hat{O}_{2} \mid \Omega_{\tilde{T}_{1}} F\right\rangle .
\end{aligned}
$$

Because by definition, $\left|\Omega_{\tilde{T}_{1}} \Omega_{\tilde{T}_{2}} F\right\rangle$ and $\left|\Omega_{\tilde{T}_{2}} \Omega_{\tilde{T}_{1}} F\right\rangle$ can at most differ by a phase factor, which is just $\eta$ :

$$
\left|\Omega_{\tilde{T}_{1}} \Omega_{\tilde{T}_{2}} F\right\rangle=\eta\left|\Omega_{\tilde{T}_{2}} \Omega_{\tilde{T}_{1}} F\right\rangle .
$$


Namely $\eta$ can be computed as the phase of the following product:

$$
\begin{gathered}
\eta \sim\left\langle F\left|\hat{O}_{2}^{-1}\right| \Omega_{\tilde{T}_{2}} F\right\rangle \cdot\left\langle\left.\Omega_{\tilde{T}_{2}} F\right|^{\tilde{T}_{2}}\left(\hat{O}_{1}^{-1}\right) \mid \Omega_{\tilde{T}_{1}} \Omega_{\tilde{T}_{2}} F\right\rangle \\
\cdot\left\langle\left.\Omega_{\tilde{T}_{1}} \Omega_{\tilde{T}_{2}} F\right|^{\tilde{T}_{1}} \hat{O}_{2} \mid \Omega_{\tilde{T}_{1}} F\right\rangle \cdot\left\langle\Omega_{\tilde{T}_{1}} F\left|\hat{O}_{1}\right| F\right\rangle
\end{gathered}
$$

The advantage of this expression of $\eta$ is that it is explicitly independent of the global phase choices of involved four fluxon states. Although Eq. (C7) holds for arbitrary operators $\hat{O}_{i}$ satisfying Eq. C4 , there exist particularly convenient choices of these operators. Let us choose $\hat{O}_{i}=\hat{O}_{i}^{a d}$ as the adiabatic transporting of the unit fluxon $|F\rangle$ from its original position by one lattice spacing along the $\vec{a}_{i}$ direction $(i=1,2)$. Precisely, $\hat{O}_{i}^{a d}$ are defined as the following time-revolutions:

$$
\hat{O}_{i}^{a d}=W_{i} \cdot \mathcal{T} \exp \left(-i \int_{0}^{T} d t H_{i}(t)\right),
$$

where $\mathcal{T}$ is the time ordering. Starting from the original Hamiltonian $H_{\text {orig }}, H_{i}(0) \equiv H_{0}$ is the modified Hamiltonian whose ground state hosts a fluxon $|F\rangle$ located in the region $D$ (and another anti-fluxon far away from $D$ ). $W_{i} H_{i}(T) W_{i}^{-1}={ }^{\Omega_{\tilde{T}_{i}}} H_{0}$ which hosts the fluxon- $F$ in $D$ spatially translated by one lattice spacing (but the antifluxon is not movied), and $W_{i}$ is the local gauge transformation near $F$ needed in order to make $H_{i}(t)$ a smooth time-evolution.

Here the definition of ${ }^{\Omega_{\tilde{T}_{i}}} H_{0}$ is the following: for those terms of $H_{0}$ near the location of the fluxon $F$, they are conjugated by the global operation $\tilde{T}_{i}$; while all other terms stay invariant as in $H_{0}$. Because inside region $D$, $H_{0}$ is different from $H_{\text {orig }}$ only by those terms near the location of $F$, and $H_{\text {orig }}$ is $\tilde{T}_{i}$ symmetric, such a definition is self-consistent. Clearly, $\left|\Omega_{\tilde{T}_{i}} F\right\rangle$ is the ground state of $\Omega_{\tilde{T}_{i}} H_{0}$. Note that ${ }^{\Omega_{\tilde{T}_{1}}} \Omega_{\tilde{T}_{2}} H_{0}={ }_{\tilde{T}_{2}} \Omega_{\tilde{T}_{1}} H_{0}$ (due to the fact that every local term of Hamiltonian need to be $U(1)$ symmetric) and $\left|\Omega_{\tilde{T}_{1}} \Omega_{\tilde{T}_{2}} F\right\rangle$ is its ground state.

We will adopt the notation ${ }^{\Omega_{\tilde{T}_{i}}} \hat{S}$ for a similarly defined transformation of a general operator $\hat{S}$ which is a summation of many local terms, which requires that within $D,{ }^{\tilde{T}} i \hat{S}-\hat{S}$ is composed of terms only near the location of the fluxon $F$. After discretizing the time-evolution then considering the limit of the time-step going to zero, it is straightforward to establish that:

$$
\begin{aligned}
& \left\langle\Omega_{\tilde{T}_{1}} F\left|\hat{O}_{1}^{a d}\right| F\right\rangle=\left\langle\left.\Omega_{\tilde{T}_{2}} \Omega_{\tilde{T}_{1}} F\right|^{\Omega_{\tilde{T}_{2}}} \hat{O}_{1}^{a d} \mid \tilde{T}_{2} F\right\rangle, \\
& \left\langle\Omega_{\tilde{T}_{2}} F\left|\hat{O}_{2}^{a d}\right| F\right\rangle=\left\langle\left.\Omega_{\tilde{T}_{1}} \Omega_{\tilde{T}_{2}} F\right|^{\Omega_{\tilde{T}_{1}}} \hat{O}_{2}^{a d} \mid \tilde{T}_{1} F\right\rangle,
\end{aligned}
$$

where ${ }^{\Omega_{\tilde{T}_{j}}} \hat{O}_{i}^{a d}={ }^{T_{j}} W_{i} \cdot \mathcal{T} \exp \left(-i \int_{0}^{T} d t^{\Omega_{\tilde{T}_{j}}} H_{i}(t)\right)$. Note that because gauge transformations commute with each other, we have: ${ }^{\widetilde{T}_{j}} W_{i}={ }^{T_{j}} W_{i}$; namely the conjugate of magnetic translation on gauge transformation is just as the usual translation. This identity is eventually related to the fact that the magnetic translation algebra of the unit fluxon, just as the usual translation algebra, measures $e^{2 \pi i \bar{\rho}}$.
Therefore, $\eta$ can be computed as:

$$
\begin{aligned}
\eta \sim\langle F| \mathcal{T} \exp \left(i \int_{-T}^{0} d t H_{2}(-t)\right) \cdot W_{2}^{-1} \\
\cdot \mathcal{T} \exp \left(i \int_{-T}^{0} d t^{\Omega_{\tilde{T}_{2}}} H_{1}(-t)\right) \cdot{ }^{T_{2}} W_{1}^{-1} \\
.{ }^{T_{1}} W_{2} \cdot \mathcal{T} \exp \left(-i \int_{0}^{T} d t^{\Omega_{\tilde{T}_{1}}} H_{2}(t)\right) \\
\cdot W_{1} \cdot \mathcal{T} \exp \left(-i \int_{0}^{T} d t H_{1}(t)\right)|F\rangle \\
=\langle F| W_{2}^{-1 T_{2}} W_{1}^{-1 T_{1}} W_{2} W_{1} \\
. W_{1}^{-1 T_{1}} W_{2}^{-1 T_{2}} W_{1} W_{2}\left[\mathcal{T} \exp \left(i \int_{-T}^{0} d t H_{2}(-t)\right)\right] \\
. W_{1}^{-1 T_{1}} W_{2}^{-1 T_{2}} W_{1}\left[\mathcal{T} \exp \left(i \int_{-T}^{0} d t^{\Omega_{\tilde{T}_{2}}} H_{1}(-t)\right)\right] \\
. W_{1}^{-1}\left[\mathcal{T} \exp \left(-i \int_{0}^{T} d t^{\Omega_{\tilde{T}_{1}}} H_{2}(t)\right)\right] \\
. \mathcal{T} \exp \left(-i \int_{0}^{T} d t H_{1}(t)\right)|F\rangle \\
\equiv\left\langle F\left|W_{2}^{-1 T_{2}} W_{1}^{-1 T_{1}} W_{2} W_{1} \cdot \hat{O}_{d}^{a d} \hat{O}_{l}^{a d} \hat{O}_{u}^{a d} \hat{O}_{r}^{a d}\right| F\right\rangle
\end{aligned}
$$

After the second equation mark, we use accumulated gauge transformations to conjugate the time-evolutions. After this treatment, the gauge during the whole fourstep time-evolution is smooth, and we are only left with the final gauge transformation $W_{2}^{-1 T_{2}} W_{1}^{-1} T_{1} W_{2} W_{1}$, which is easy to compute. After the third equation mark, we reserve the symbols $\hat{O}_{d}^{a d}, \hat{O}_{l}^{a d}, \hat{O}_{u}^{a d}, \hat{O}_{r}^{a d}$ to describe the adiabatic time-evolutions for the right,up, left and down move in this smooth gauge.

Next we compute this phase $\eta$ explicity by perturbation theory. The basic idea is to choose the gauge in $H_{1}(t), H_{2}(t)$ so that they are only perturbatively different from $H_{0}$. We choose a rectangular region on a square lattice to demonstrate the calculation. Let $(x, y)$ be the coordinate of a site. The adjacent sites are connected by links carrying the gauge field: $A_{x}(x, y)$ connect $(x, y)$ to $(x+1, y)$, and $A_{y}(x, y)$ connect $(x, y)$ to $(x, y+1)$. Consider a rectangular region: $0 \leq x \leq L_{x}, 0 \leq y \leq L_{y}$ which contains $L_{x} L y$ plaquettes. Initially, the state $|F\rangle$ is the ground state of $H_{0}$ hosting a single flux uniformly distributed in this region (i.e. every plaquette hosts $\varphi \equiv \frac{2 \pi}{L_{x} L_{y}}$ flux), the corresponding gauge field configuration are denoted as $A_{x, y}^{0}(x, y)$. One can choose a gauge to describe this flux distribution. Then, we specifically define $H_{i}(t)(0 \leq t \leq 1)$ as:

$$
\begin{aligned}
& H_{1}(t)=H_{0}\left(\left\{A_{x}^{0}(x, y), A_{y}^{0}(x, y)+\delta A_{y}(x, y, t)\right\}\right), \\
& H_{2}(t)=H_{0}\left(\left\{A_{x}^{0}(x, y)+\delta A_{x}(x, y, t), A_{y}^{0}(x, y)\right\}\right),
\end{aligned}
$$

where $\delta A_{y}(x, y, t)=-\varphi(t)$ if $1 \leq x \leq L_{x}, 0 \leq y \leq L_{y}-1$, and zero otherwise, $\varphi(t=0)=0$ and $\varphi(t=T)=\varphi$. And $\delta A_{y}(x, y, t)=\varphi(t)$ if $0 \leq x \leq L_{x}-1,1 \leq y \leq L_{y}$. 
Namely, we choose a convenient gauge to describe the time-evolutions. When the region is very large, $\varphi$ goes to zero. We will use $\varphi$ as the perturbative parameter to control the calculation.

We will only keep the leading order in our perturbative calculation. Taylor expanding these time-dependent Hamiltonians lead to:

$$
\begin{aligned}
& H_{1}(t)=H_{0}-\varphi(t) \sum_{1 \leq x \leq L_{x}}^{0 \leq y \leq L_{y}-1} j_{y}(x, y)+O\left(\varphi^{2}\right), \\
& H_{2}(t)=H_{0}+\varphi(t) \sum_{0 \leq x \leq L_{x}-1}^{1 \leq y \leq L_{y}} j_{x}(x, y)+O\left(\varphi^{2}\right) \text {, }
\end{aligned}
$$

where the current operators living on links are obtained by partial differentiating $H_{0}$ w.r.t. the corresponding gauge fields. It then follows that to the leading order of $\varphi$ :

$$
\begin{aligned}
\hat{O}_{r / u}^{a d} & =\mathcal{T} \exp \left[-i \int_{0}^{T} d t\left(H_{0}+O(\varphi) \sum_{0 \leq x \leq L_{x}}^{0 \leq y \leq L_{y}} j_{x}\right.\right. \\
& \left.\left.+O(\varphi) \sum_{0 \leq x \leq L_{x}}^{0 \leq y \leq L_{y}} j_{y}\right)\right], \\
\hat{O}_{l / d}^{a d} & =\mathcal{T} \exp \left[+i \int_{0}^{T} d t\left(H_{0}+O(\varphi) \sum_{0 \leq x \leq L_{x}}^{0 \leq y \leq L_{y}} j_{x}\right.\right. \\
& \left.\left.+O(\varphi) \sum_{0 \leq x \leq L_{x}}^{0 \leq L_{y}} j_{y}\right)\right] .
\end{aligned}
$$

Because we are considering a charge insulator, the spatial summation current density is zero in the ground state of $H_{\text {orig. }}$. Due to the extra factor $O(\varphi)$, it is safe to ignore these current contributions to the leading order of $\varphi$. We conclude that to the leading order of $\varphi, \eta$ is due to the following phase:

$$
\eta=\left\langle F\left|W_{2}^{-1 T_{2}} W_{1}^{-1 T_{1}} W_{2} W_{1}\right| F\right\rangle,
$$

where $W_{2}^{-1 T_{2}} W_{1}^{-1 T_{1}} W_{2} W_{1}$ is the required gauge transformation to send the final Hamiltonian $H_{\text {final }}$ in the smooth gauge (i.e., the Hamiltonian after $O_{d}^{a d}$ ) back to the original gauge of $H_{0}$. Note that after $O_{r}^{a d}$, $H=H_{0}-\varphi \sum_{1 \leq x \leq L_{x}}^{0 \leq y \leq L_{y}-1} j_{y}(x, y)$. After $O_{u}^{a d}, H=H_{0}-$ $\varphi \sum_{1 \leq x \leq L_{x}}^{0 \leq y \leq L_{y}-1} j_{y}(x, y)+\varphi \sum_{1 \leq x \leq L_{x}}^{1 \leq y \leq L_{y}} j_{x}(x, y)$. After $O_{l}^{a d}$, $H=H_{0}-\varphi \sum_{1 \leq x \leq L_{x}}^{0 \leq y \leq L_{y}-1} j_{y}(x, y)+\varphi \sum_{1 \leq x \leq L_{x}}^{1 \leq y \leq L_{y}} j_{x}(x, y)+$ $\varphi \sum_{1 \leq x \leq L_{x}}^{1 \leq y \leq L_{y}} j_{y}(x, y)$. Therefore, after $O_{d}^{a \bar{d}}$,

$$
\begin{aligned}
H_{\text {final }} & =H_{0}-\varphi \sum_{1 \leq x \leq L_{x}}^{0 \leq y \leq L_{y}-1} j_{y}(x, y)+\varphi \sum_{1 \leq x \leq L_{x}}^{1 \leq y \leq L_{y}} j_{x}(x, y) \\
& +\varphi \sum_{1 \leq x \leq L_{x}}^{1 \leq y \leq L_{y}} j_{y}(x, y)-\varphi \sum_{0 \leq x \leq L_{x}-1}^{1 \leq y \leq L_{y}} j_{x}(x, y) \\
& =H_{0}-\varphi \sum_{1 \leq x \leq L_{x}}^{1 \leq y \leq L_{y}} j_{y}(x, y)+\varphi \sum_{x=L_{x}}^{1 \leq j_{x}(x, y)} \\
& +\varphi \sum_{1 \leq x \leq L_{x}} j_{y}(x, y)-\varphi \sum_{x=0}^{1 \leq y \leq L_{y}} j_{x}(x, y) . \quad(\mathrm{C} 15)
\end{aligned}
$$

Note that the current terms form a loop enclosing sites $1 \leq x \leq L_{x}, 1 \leq y \leq L_{y}$. Clearly, the required gauge transformation is the following:

$$
\begin{aligned}
\eta & =\left\langle F\left|W_{2}^{-1 T_{2}} W_{1}^{-1 T_{1}} W_{2} W_{1}\right| F\right\rangle \\
& =\left\langle F\left|\exp \left[i \varphi \sum_{1 \leq x \leq L_{x}}^{1 \leq y \leq L_{y}} \hat{n}(x, y)\right]\right| F\right\rangle
\end{aligned}
$$

where $\hat{n}(x, y)$ is the particle number operator on site $(x, y)$. Because $\varphi=2 \pi /\left(L_{x} L_{y}\right)$, this is exactly measuring the average charge density per unit cell: $e^{2 \pi i \bar{\rho}}$.
[1] K. v. Klitzing, G. Dorda, and M. Pepper, Phys. Rev. Lett. 45, 494 (1980)

[2] D. C. Tsui, H. L. Stormer, and A. C. Gossard, Phys. Rev. Lett. 48, 1559 (1982)

[3] X.-G. Wen, Quantum Field Theory Of Many-body Systems: From The Origin Of Sound To An Origin Of Light And Electrons (Oxford University Press, New York, 2004).

[4] D. R. Hofstadter, Phys. Rev. B 14, 2239 (1976)

[5] D. J. Thouless, M. Kohmoto, M. P. Nightingale, and M. den Nijs, Phys. Rev. Lett. 49, 405 (1982)

[6] M. Kohmoto, Annals of Physics 160, 343 (1985)

[7] Q. Niu, D. J. Thouless, and Y.-S. Wu, Phys. Rev. B 31, $3372(1985)$
[8] F. D. M. Haldane, Phys. Rev. Lett. 61, 2015 (1988).

[9] K. Sun, Z. Gu, H. Katsura, and S. Das Sarma, Phys. Rev. Lett. 106, 236803 (2011).

[10] E. Tang, J.-W. Mei, and X.-G. Wen, Phys. Rev. Lett. 106, 236802 (2011)

[11] T. Neupert, L. Santos, C. Chamon, and C. Mudry, Phys. Rev. Lett. 106, 236804 (2011).

[12] S. A. Parameswaran, R. Roy, and S. L. Sondhi, Topological insulators / Isolants topologiques Topological insulators / Isolants topologiques, Comptes Rendus Physique 14, $816(2013)$

[13] E. Lieb, T. Schultz, and D. Mattis, Annals of Physics 16, 407 (1961).

[14] M. Oshikawa, M. Yamanaka, and I. Affleck, Phys. Rev. 
Lett. 78, 1984 (1997)

[15] M. Oshikawa, Phys. Rev. Lett. 84, 1535 (2000).

[16] M. B. Hastings, EPL (Europhysics Letters) 70, 824 (2005)

[17] H. C. Po, H. Watanabe, M. P. Zaletel, and A. Vishwanath, Science Ad$\begin{array}{llll}\text { vances } & \mathbf{2} & \text { (2016), } & 10.1126 / \text { sciadv.1501782, }\end{array}$ http://advances.sciencemag.org/content/2/4/e1501782.full.p

[18] J. Zak, Phys. Rev. 134, A1602 (1964).

[19] J. Zak, Phys. Rev. 134, A1607 (1964).

[20] I. Dana, Y. Avron, and J. Zak, Journal of Physics C: Solid State Physics 18, L679 (1985).

[21] J. E. Avron and L. G. Yaffe, Phys. Rev. Lett. 56, 2084 (1986)

[22] A. Kol and N. Read, Phys. Rev. B 48, 8890 (1993).

[23] A. M. Essin and M. Hermele, Phys. Rev. B 87, 104406 (2013)

[24] M. Barkeshli, P. Bonderson, M. Cheng, and Z. Wang, ArXiv e-prints (2014), arXiv:1410.4540 [cond-mat.str-el]

[25] M. B. Hastings, Phys. Rev. B 69, 104431 (2004).

[26] M. Oshikawa and T. Senthil, Phys. Rev. Lett. 96, 060601 (2006)

[27] M. Cheng, M. Zaletel, M. Barkeshli, A. Vishwanath, and P. Bonderson, Phys. Rev. X 6, 041068 (2016)

[28] M. Oshikawa, Phys. Rev. Lett. 84, 3370 (2000).

[29] M. Oshikawa and T. Senthil, Phys. Rev. Lett. 96, 060601 (2006).

[30] R. Tao and Y.-S. Wu, Phys. Rev. B 30, 1097 (1984).

[31] X.-G. Wen, Advances in Physics 44, 405 (1995).

[32] K. Fujikawa and H. Suzuki, Path Integrals and Quantum Anomalies, reprint edition ed., International Series of Monographs on Physics (Oxford University Press, 2014).

[33] R. Resta, Rev. Mod. Phys. 66, 899 (1994).

[34] R. Resta, Journal of Physics: Condensed Matter 14, R625 (2002)

[35] R. Resta and S. Sorella, Phys. Rev. Lett. 82, 370 (1999).

[36] I. Souza, T. Wilkens, and R. M. Martin, Phys. Rev. B 62, $1666(2000)$

[37] R. Resta, Journal of Physics: Condensed Matter 14, R625 (2002).

[38] M. Nakamura and J. Voit, Phys. Rev. B 65, 153110 (2002).

[39] A. A. Aligia and G. Ortiz, Phys. Rev. Lett. 82, 2560 (1999).

[40] R. Tao and D. J. Thouless, Phys. Rev. B 28, 1142 (1983)

[41] A. Seidel, H. Fu, D.-H. Lee, J. M. Leinaas, and J. Moore, Phys. Rev. Lett. 95, 266405 (2005).

[42] R. B. Laughlin, Phys. Rev. B 23, 5632 (1981).

[43] X. G. Wen and Q. Niu, Phys. Rev. B 41, 9377 (1990).
[44] N. Tarantino, N. H. Lindner, and L. Fidkowski, New Journal of Physics 18, 035006 (2016).

[45] A. Kitaev, January Special Issue, Annals of Physics 321, $2(2006)$

[46] X.-G. Wen, Phys. Rev. Lett. 90, 016803 (2003).

[47] M. Barkeshli and X.-L. Qi, Phys. Rev. X 2, 031013 (2012)

pł⿷3] S. Jiang and Y. Ran, Phys. Rev. B 95, 125107 (2017).

[49] M. S. Dresselhaus, G. Dresselhaus, and A. Jorio, Group Theory: Application to the Physics of Condensed Matter (Springer-Verlag Berlin Heidelberg, 2008).

[50] S. A. Parameswaran, A. M. Turner, D. P. Arovas, and A. Vishwanath, Nat Phys 9, 299 (2013)

[51] P. G. Harper, Proceedings of the Physical Society. Section A 68, 879 (1955).

[52] M. Bercx, M. Hohenadler, and F. F. Assaad, Phys. Rev. B 90, 075140 (2014)

[53] Y.-C. He, S. Bhattacharjee, R. Moessner, and F. Pollmann, Phys. Rev. Lett. 115, 116803 (2015).

[54] I. Martin and C. D. Batista, Phys. Rev. Lett. 101, 156402 (2008).

[55] T. Li, EPL (Europhysics Letters) 97, 37001 (2012).

[56] V. Kalmeyer and R. B. Laughlin, Phys. Rev. Lett. 59, 2095 (1987)

[57] A. Y. Kitaev, Annals of Physics 303, 2 (2003).

[58] T. Senthil, ArXiv e-prints (2014), arXiv:1405.4015 [condmat.str-el] .

[59] A. Matsugatani, Y. Ishiguro, K. Shiozaki, and H. Watanabe, Phys. Rev. Lett. 120, 096601 (2018)

[60] F. Wilczek and A. Zee, Phys. Rev. Lett. 51, 2250 (1983)

[61] A. S. GOLDHABER, R. MACKENZIE, and F. WILCZEK, Modern Physics Letters A, Mod. Phys. Lett. A 04, 21 (1989).

[62] In fact, as is well known, quantization of the Hall conductivity in a realistic setting requires existence of localized states due to impurities. These localized states generally give "gapless" excitations in terms of energy spectrum, but they do not respond to flux insertion and do not contribute to the Hall current. In such a circumstance, we may extend the notion of "gapped" system from a system with a non-vanishing gap to extended states. This subtlety is inherited from Laughlin's original argument to the present version without edge states. On the (mathematically) safe side, we can discuss the Hall conductivity simply assuming a gap, even though this does not directly address experimentally observed Quantum Hall Effects. This is indeed a nontrivial and interesting question, as we can see from Thouless-Kohmoto-Nightingale-den Nijs paper and its subsequent generalizations. 